\title{
Overview of APEX Project Results
}

\author{
Lubomir Prech $^{1 *}$, Yuri Y. Ruzhin ${ }^{2}$, Vladimir S. Dokukin ${ }^{2}$, Zdenek Nemecek $^{1}$ and \\ Jana Safrankova ${ }^{1}$
}

${ }^{1}$ Faculty of Mathematics and Physics, Charles University, Prague, Czechia, ${ }^{2}$ Pushkov Institute of Terrestrial Magnetism, Ionosphere and Radiowave Propagation (IZMIRAN), Russian Academy of Sciences, Troitsk, Russia

OPEN ACCESS

Edited by:

Gian Luca Delzanno,

Los Alamos National Laboratory

(DOE), United States

Reviewed by:

Geoffrey D. Reeves, Los Alamos National Laboratory

(DOE), United States Harald Uwe Frey, University of California, Berkeley, United States

*Correspondence: Lubomir Prech lubomir.prech@mff.cuni.cz

Specialty section:

This article was submitted to Space Physics, a section of the journal Frontiers in Astronomy and Space

Sciences

Received: 30 September 2018 Accepted: 06 December 2018 Published: 21 December 2018

Citation:

Prech L, Ruzhin YY, Dokukin VS, Nemecek Z and Safrankova J (2018)

Overview of APEX Project Results. Front. Astron. Space Sci. 5:46. doi: 10.3389/fspas.2018.00046
The APEX mother-daughter project (Active Plasma EXperiments) was launched into an elliptical polar orbit (440-3,080 km) in December 1991. It consisted of the main Russian Interkosmos-25 (IK-25) satellite and the Czech MAGION-3 subsatellite, both with international scientific payloads. The mission used intensive modulated electron beam emissions and xenon plasma or neutral releases from the main satellite for studies of dynamic processes in the magnetosphere and upper ionosphere. Its main scientific objectives were to simulate an artificial aurora and to study optical and radio emissions from the aurora region, and to investigate the dynamics and relaxation of modulated electron and plasma jets, artificially injected into the ionospheric plasma. The experiments studied the Critical Ionization Velocity phenomenon and a diamagnetic cavity formation during the xenon releases, local and distant effects of the electron beam injection, spacecraft charging and potential balance, and plasma-wave interactions during the artificial emissions. Attempts were performed to utilize the modulated electron beam as an active transmitting antenna in the space. The theory of ballistic wave propagation across plasma barrier was tested in a joint active experiment with the Dushanbe ionospheric heater facility. In the paper, we give a short overview of the IK-25/MAGION-3 scientific instrumentation and methodology of experiments with artificial beam injections and we provide a review of the main APEX active experiments results, many of which have been published only in the Russian language so far. From a historical 25-years-long perspective, we try to put the results of the APEX experiments into the context of other active experiments in the space plasma.

Keywords: active plasma experiment in space, electron beam, xenon plasma injection, neutral xenon injection, plasma waves, APEX, INTERCOSMOS-25, MAGION-3

\section{INTRODUCTION}

Active experiments in space plasma utilize many different agents to disturb the ionospheric or magnetospheric environment and to stimulate many variable but rather rare natural phenomena under controlled conditions (Raitt, 1995). The response is then studied in order to get information on natural space structures (e.g., electron beam tracing of magnetospheric or ionospheric electric and magnetic fields), to use artificially produced phenomena as models of natural ones (artificial aurora etc.) or to verify mechanisms which would explain some physical processes (e.g., beam-plasma interactions, wave generation, photochemical reactions) when the relevant physical conditions cannot be achieved in laboratory experiments. Some active experiments have close connections to technology. They are motivated by problems in the design and reliability of the artificial satellites or they help to develop new power generators or propulsion methods. 
Since 1960s, tens of active charged particle beam experiments (e.g., Winckler, 1980; Grandal, 1982; Podgorny, 1982; Neubert and Banks, 1992; Raitt, 1995), in space have been performed, the motivations behind these experiments ranging from investigations of charge neutralization processes in the plasma medium near the beam source to probing conditions on remote sections of geomagnetic field lines (Winckler, 1980). A lot of interesting experimental results was obtained aboard the U.S. space shuttles (e.g., Burch, 1986), few other satellites were devoted to active experiments in space plasma at altitudes starting in ionosphere to solar wind. During last two decades their frequency has decreased and the scientific community has turned more to theoretical studies, computer simulations, or data reanalysis in this field. New science mission concepts (e.g., MacDonald et al., 2012) may return active space plasma experiment to the foreground of scientific interest.

The topic of this overview paper-the APEX project satellite pair-had relatively unique orbit among the active experiment spacecraft as it will be described later. It had extensive scientific payload and ambitious science plans. Although it is more than 26 years since the launch now, new original research articles still profit from the collected data. We bring an overview of the active APEX experiments and their results, while many other interesting results of passive ionospheric observations (often based on the two-point satellite measurements rare in that era) are out of the scope of this paper. The overview should not be considered as a complete list of references to the APEX project related literature, some results were originally published in Russian and only later appeared in a Russian translation version of the journal or in other English language journals or proceedings and for such cases we usually cite just one reference.

The review is organized to several sections. The first one comprises introduction to the APEX project, its origin, scientific goals, and a short summary of the scientific instruments onboard the two satellites of the project. Comments to the active experiment planning and telemetry issues are included here as well. The result overview section starts with analysis of the main satellite charging during the passive observations and active experiments with the electron beam injection neutralized by neutral xenon/xenon plasma releases or during the xenon plasma releases itself. Overview of the neutral xenon releases related to the critical ionization velocity (CIV) phenomenon processes follows. Next part is devoted the beam-plasma interaction, discussing the plasma environment in a vicinity of the main satellite during the beam injections, diamagnetic cavity formation, electron pitch-angle distributions, and generation of ELF/VLF and HF waves. Distant injection effects as observed from the subsatellite at distances of hundreds of kilometers are summarized in the next subsection. The last part briefly covers the ionospheric heating experiments in which the main APEX satellite participated. Summary and concluding remarks close the article.

\section{APEX PROJECT}

The international experiment APEX in frame of the former INTERCOSMOS science program was suggested by IZMIRANthe Pushkov Institute of Terrestrial Magnetism, Ionosphere, and Radio Wave Propagation of the Russian Academy of Sciences, Troitsk, Russia, and the Geophysical Institute of Czechoslovak Academy of Sciences, Prague, Czech Republic (GFI, the group is affiliated to the Institute of Atmospheric Physics of Czech Academy of Sciences today) in mid 1980's. The project followed previous Soviet/international active experiments with sounding rockets and satellites (Stereotop, PORCUPINE, Trigger, Zarnitsa, G-60-S, ARAKS, ACTIVE) (Cambou et al., 1975, 1980; Dokukin et al., 1981; Haerendel and Sagdeev, 1981; Sagdeev et al., 1981; Managadze et al., 1988; Klos et al., 1998), this time in a new mother-daughter configuration and with wide international payload (Russia, Ukraine, Czechoslovakia, Poland, Rumania, Bulgaria, Hungary, eastern Germany). The name of the Interkosmos programme project APEX is abbreviated from from words “Active Plasma EXperiments." The core of the project were active experiments with injection of electron and (or) plasma beams from a board of the low Earth's orbit (LEO) satellite with simultaneous registration of the phenomena, produced by interaction of injected beams with background plasma.

Essentially important peculiarity of the project were synchronous measurements of the basic physical parameters of the environment, of the beam and generated fields on two spaceseparated vehicles-the main satellite APEX Interkosmos-25 and its subsatellite MAGION-3 (Figure 1). The independent subsatellite with small gas trusters for orbit correction carried out simultaneous measurements on various mutual distances (from $10 \mathrm{~m}$ up to $1,000-2,000 \mathrm{~km}$ ), at different areas of beam-disturbed environment, at different zones of the Earth's magnetosphere and ionosphere. The "boomerang" maneuvers of MAGION-3 were performed few times: it was pushed forward along the orbit and returned backward to main satellite, so MAGION-3 escaped from the main IK-25 up to few hundreds $\mathrm{km}$ and came back at distance about $0.4 \mathrm{~km}$ (Figure 2). An important part of the program were investigations of natural and human-made phenomena in a passive mode. The presence of two spacecrafts, equipped with practically equivalent complexes of the scientific equipment, enabled not only to carry out diverse researches of the ionosphere and the bottom magnetosphere, but also to distinguish spatial and temporal structures of the observable phenomena. A nearly polar orbit allowed to carry out research in auroral area. The complex program of "under satellite" ground observation and experiments included standard geophysical monitoring and special programs with ionospheric heating facilities.

Both spacecrafts, the main IK-25 and its subsatellite MAGION-3, were launched on an elliptical orbit with apogee of $3,080 \mathrm{~km}$ and perigee of $440 \mathrm{~km}$, with the $82.5^{\circ}$ inclination of the orbital plane on December 18, 1991 from the Russian space base Plesetsk. Orbital period was $\sim 2$ hours.

\subsection{APEX Scientific Goals}

The scientific goals of the project as described in Oraevsky et al. (1992) and Oraevsky and Triska (1993) are summarized below:

- Study of the dynamics and relaxation of modulated electron and plasma jets artificially injected into near-Earth plasma.

- Study of the low-frequency and MHD waves generation by heavy ions in the ionosphere. 
- Determination of the radio emission generated by modulated beams of charged particles.

- Search for non-linear wave structures of the electromagnetic soliton type in a disturbed plasma environment.

- Study of the process of neutralization and dynamics of spacecraft charging.

- Study of the nature of the electrodynamic relationship between electromagnetic waves in the magnetosphere and ionosphere.

- Study of optical and radio emission in the auroral region.

- Passive mode observations-ionospheric plasma profiles under different conditions, auroral precipitation dynamics, mapping, and dynamics of polar cusps etc.

The APEX programme principal investigator (PI) was Viktor N. Oraevsky (1935-2006) from IZMIRAN, the MAGION subsatellite programme founder and PI was Pavel Triska (19312018) from GFI.

\subsection{Scientific Payload}

The main satellite IK-25 design was based on the AUOS-Z-AP-IK spacecraft bus aboard of the Tsyklon-3 rocket launcher, both produced by the KB Yuzhnoe, Dnepropetrovsk, Ukraine. The MAGION-3 subsatellite platform was designed and produced by the Geophysical Institute in Prague.

Both satellites were equipped with a complex scientific payload developed in a wide international cooperation. The particle, fields, wave, and supporting diagnostics are enumerated in Tables 1, 2.

\subsubsection{IK-25 Short Description}

The AUOS-Z bus consisted of a pressurized cylinder of about $1 \mathrm{~m}$ diameter and height $\sim 2 \mathrm{~m}$. Eight solar panels $\left(\sim 12 \mathrm{~m}^{2}\right)$ were deployed $30^{\circ}$ away (petal-like). The IK-25 satellite was gravitationally stabilized, its vertical position being kept by a spherical weight on about $20 \mathrm{~m}$ long boom (see Figure 1). The spacecraft utilized the $28 \mathrm{~V}$ power bus with the + pole commutation. Spacecraft charging characteristics (see section 3.1.1) suggested the solar panels were partially exposed to the ionospheric plasma. The current collection through the solar panel area in contact with the plasma depends on the panel design and technology-individual solar cell interconnects and edges exposure, coverglass conductivity/presence of a grounded transparent conductive coating, potential bias between the solar cells/cell interconnect layout etc., but such details of the AUOS-Z bus are unknown to us. For an example of the recent solar panel current collection modeling (see, e.g., Hess et al., 2016).

The UEM-2 electron gun consisted of two electron injectors G1 (oriented opposite to the satellite orbital velocity vector) and G2 (inclined toward the Earth). Utilizing a magnetic deflector, the G2 injector could turn the electron beam to the G1 beam orientation (see Figure 3) that corresponded to the injection pitch angles $50-80^{\circ}$ for typical active parts of the IK-25 orbit. The electron acceleration voltage could reach $8-10 \mathrm{kV}$ (unstabilized) with the peak current $\sim 30,70$, or $100 \mathrm{~mA}$. The initial beam width was $4 \mathrm{~mm}$ at the output aperture. The electron gun could run in two basic modulation modes:
- Fixed frequencies: F40K (40 kHz), F15.6K, F976-all $2 \mu \mathrm{s}$ pulses, F3906, F30.5 (32 $\mu$ s pulses)

- Sweeping frequency: S250 $(30.5 \mathrm{~Hz}-250 \mathrm{kHz}, 2 \mu \mathrm{s}$ pulses, $1 \mathrm{DC}$ +11 frequency steps $-1 s$ each) and $\mathrm{S} 15.6(30.5 \mathrm{~Hz}-15.6 \mathrm{kHz}$, $32 \mu$ s pulses, $1 \mathrm{DC}+11$ frequency steps $-1 s$ each), basic cycle length $23 s$

The UPM xenon plasma injector utilized a stationary plasma thruster (SPT) of Hall type (with close electron drift and extended acceleration zone) (Artsimovich et al., 1974) similar as used in the PORCUPINE experiment (Haerendel and Sagdeev, 1981). The xenon plasma was released to a $60^{\circ}$ wide cone oriented $\sim 45^{\circ}$ from the zenith direction as illustrated in Figure 3. The ion energy reached 200-250 $\mathrm{eV}$, ion temperature $\sim 50 \mathrm{eV}$, ion current $2 A\left(10^{19}\right.$ ions/s), $100 \%$ ionization degree. As only xenon ions can leave the main discharge and accelerator section of the thruster, electrons from a supplementary xenon discharge (two external hollow cathodes $\mathrm{LaB}_{6}$ installed, electron temperature $\sim 2 \mathrm{eV}$ ) initiated the main discharge and simultaneously neutralized the xenon plasma beam injected to the space. The injector could work in these modes:

- Neutral xenon release, $\sim 3 \mathrm{mg} / \mathrm{s}$,

- DC xenon plasma emission-F0

- Modulated xenon plasma emission (almost 100\% current modulation)-F1000 (1 kHz; 62, 125, 250, and $500 \mathrm{~Hz}$ modulation frequencies were other options)

The UPM cycle consisted of $\sim 35-45 s$ preheating interval (with neutral xenon release lasting $\sim 5-6 s$, further denoted as Xe$\mathrm{A}$, and the hollow cathode emission, further as $\mathrm{Xe}-\mathrm{B}$ ) and one or several intervals of $\sim 100 s$ plasma injection followed by $\sim$ $50 s$ neutral xenon release only (Xe-A/Xe-B). Housekeeping and science data revealed additional $\sim 0.12 \mathrm{~Hz}$ current modulation (up to $20 \%$ ) due to unknown internal/external feedback. Only the neutral xenon gas was released during certain orbits as the ionization voltage was not applied due to technical reasons (Oraevskii et al., 1999).

Mean injection current profiles of the UEM-2 and UPM devices are displayed in Figure 4. The UEM-2 and UPM working cycles were not synchronized, one active experiment lasted usually $\sim 3-5 \mathrm{~min}$. Emission current and acceleration voltage (housekeeping) data were averaged over 0.32 or $0.08 s$ intervals. The UEM-2 and KM-10 floating probe telemetry data indicate a low intensity electron beam was also present during the onesecond gaps between the modulated beam injections and in time between the S250 modulation sequences.

The KM-10 cold plasma monitor (also described in Afonin et al., 1994) consisted of the sensor and main electronics boxes. The sensor box was mounted on a $\sim 0.8 \mathrm{~m}$ boom in front of the solar panels in the ram direction, out of the xenon plasma/electron beams. Its box with a conductive surface was electrically isolated from the spacecraft and kept near the ionospheric plasma potential actively by means of monitoring a floating potential $\left(\Phi_{p}\right)$ of one of its 7 probes $(0.16,0.64 / 0.2$ $s$ time resolution for the RTS/STO-AP telemetries, respectively, -10 to $+16 V$ range). Ion density $N_{i}$, temperature $T_{i x}, T_{i z}$ (planar retarding potential analyzers), ion drift in the $\mathrm{YZ}$ plane, and 
TABLE 1 | IK-25 scientific payload (Dokukin, 1992).

\begin{tabular}{|c|c|}
\hline Experiment & Function \\
\hline UEM-2 electron accelerator & Injection of modulated electron beam $(30 \mathrm{~Hz}-250 \mathrm{kHz})$, more than 200 injection sessions \\
\hline UPM plasma gun & Injection of a Xe plasma beam, modulated current (DC, 32 or 1,000 Hz), more than 250 injection sessions \\
\hline KM-10 cold plasma analyzer & $\begin{array}{l}\text { Measurement of electron temperature ( } 3 \text { components), and ion drift ( } 2 \text { comp.), energy distribution, density, and temperature ( } 2 \\
\text { comp.), floating potential, } 50 \mathrm{~ms} \text { resolution }\end{array}$ \\
\hline NAM-5 mass spectrometer & $\begin{array}{l}\text { Measurement of ion composition (1-60 a.m.) of plasma by radio frequency analyses, } 3-4 \text { ion species in } 0.1 \mathrm{~s}, 8-10 \text { ion species } \\
\text { in } 0.3 \mathrm{~s}\end{array}$ \\
\hline PEAS hot electron and ion analyzer & Measurement of a energy spectrum of electrons $30 \mathrm{eV}-30 \mathrm{keV}$ in 2 planes (12 angular sectors) during $1 \mathrm{~s}$ \\
\hline DANI cold plasma complex & $\begin{array}{l}\text { Measurements of a spectrum of energies and pitch-angular distribution of low energy electrons and ions (electrostatic analyzers) } \\
\text { and parameters of plasma (Langmuir probe, Rogovsky belt) }\end{array}$ \\
\hline $\begin{array}{l}\text { NVK-ONCH low-frequency wave } \\
\text { complex }\end{array}$ & Spectra of electrical and magnetic components of low-frequency electromagnetic radiation $(0.1-20,000 \mathrm{~Hz})$ \\
\hline $\begin{array}{l}\text { VCH-VK (PRS-3) high-frequency } \\
\text { wave complex }\end{array}$ & Spectral analysis of high-frequency electromagnetic radiation, wave forms $(0.1-10 \mathrm{MHz})$ \\
\hline DEP-2 electric field experiment & Measurement of DC electric field, 3 components, 0.01-22 kHz \\
\hline SGR-5 flux-gate magnetometer & Measurement of DC magnetic field, 3 components, $\pm 64,000 n T, 0.64(0.16) s$ resolution \\
\hline ADO/service magnetometer & AUOS bus service magnetometer (attitude control), $0.64(0.16)$ and $2 s$ resolution data available \\
\hline $\begin{array}{l}\text { MNCH-2 low-frequency search coil } \\
\text { magnetometer }\end{array}$ & Measurement of low-frequency fluctuations of magnetic field, 3 components $0.1-10 \mathrm{~Hz}, 40$ or $400 \mathrm{nT}$, sensitivity $1 \mathrm{nT}$ \\
\hline FS photometer & Scanning spectrometer, 3,194-6,563 ̊ \\
\hline UF-3K 3-channel photometer & 3-channel photo-spectrometer, 8 viewing sectors, lines: 3,194, 5,577, 6,300 ̊ \\
\hline
\end{tabular}

TABLE 2 | MAGION-3 scientific payload (Triska et al., 1990).

\begin{tabular}{|c|c|}
\hline Experiment & Function \\
\hline $\begin{array}{l}\text { SGR-7/6 flux-gate magnetometer, } \\
\text { magnetic variometer }\end{array}$ & $\begin{array}{l}\text { Measurements of DC magnetic field, } 3 \text { components }( \pm 50,048 \mathrm{nT}) \text { at frequencies } 0-20 \mathrm{~Hz} \text { and } 1 \text { component }( \pm 159.4 n T) \text { at } \\
0.1-20 \mathrm{~Hz} \text { with a sensitivity } 50 \mathrm{pT}\end{array}$ \\
\hline KEM-1 ELFNLF wave experiment & $\begin{array}{l}\text { Measurements of quasi-static electric field ( } 3 \text { components) } 0.01-20 \mathrm{~Hz} \text {, electric field (2 components) } 0.1 \mathrm{~Hz}-120 \mathrm{kHz} \text {, magnetic } \\
\text { field (1 component) 10-20,000 Hz, waveforms and filter banks / spectrum analyzer }\end{array}$ \\
\hline KM-12 cold plasma analyzer & Measurement of temperature and density of cold plasma for $0.1 \mathrm{~s}$ \\
\hline ZL-A-S Langmuir probe & Electron parameter fluctuations \\
\hline PRS-2-S radio-spectrometer & Spectrum of electromagnetic waves at $0.1-10 \mathrm{MHz}$ for $0.2 \mathrm{~s} / \mathrm{spec}$.; fluctuations of magnetic field $(f<15 \mathrm{kHz})$ \\
\hline DANI-S low-energy particle analyser & Measurement of electrons and ions $0.05-1.0 \mathrm{keV}$ for $0.1 \mathrm{~s}$ \\
\hline $\begin{array}{l}\text { DOK-A-S energetic particle } \\
\text { spectrometer }\end{array}$ & $20 \mathrm{keV}-1 \mathrm{MeV}$ electron and ion fluxes, 8 energy levels, $0.1 \mathrm{~s} / \mathrm{spec}$. \\
\hline $\begin{array}{l}\text { MPS/PPS suprathermal particle } \\
\text { analyser }\end{array}$ & $\begin{array}{l}\text { Energetic spectrum of electrons } 0.05-5(20) \mathrm{keV}, 16 \text { steps, } 0.8 \mathrm{~s} / \mathrm{spec} ., 2 \text { directions; energy and angular spectrum of } \\
\text { electrons/ions } 0.02-5(20) \mathrm{keV}, 16 \text { steps, } 6 \text { angular sectors; }\end{array}$ \\
\hline FDS photometer & Measurement of optical ionospheric plasma emissions $(6,300,5,577 \AA ̊)$ \\
\hline
\end{tabular}

electron temperatures $T_{e x}, T_{e y}, T_{e z}$ (planar probes with RF bias) were the output parameters of this instrument.

The PEAS hot plasma spectrometer (Nemecek et al., 1993, 1997) measured energy-angular distributions in two planes (2 double toroidal analyzers, 12 angular sectors each, electron/positive ion sweeps alternated). Its default operational mode provided one 16-energy level spectrum $(50 \mathrm{eV}-22 \mathrm{keV})$ of electrons and ions in $2.6 s$ (STO-AP), the sampling was not synchronized with the UEM-2/UPM data sampling. The AP-1 sensor block was mounted on a one-meter boom ahead of spacecraft $\left(45^{\circ}\right.$ to $\mathrm{XZ}$ plane as depicted in Figure 1), the AP-2 sensor block was assembled $\sim 0.5 \mathrm{~m}$ above the nadir spacecraft side (XY plane), the two block distance was $\sim 3 \mathrm{~m}$. Registered particle pitch-angles (PA) distributions were computed using the $\mathrm{ADO}$ magnetometer. The block orientation corresponded to wide PA-range distributions by $\mathrm{AP}-1$ (AP-2) in auroral (equatorial) regions, respectively. During flight, several sector channels got noisy and were excluded from further data processing.

The onboard wave measurements were performed using the PRS-3 (VCH-VK complex) plasma radio spectrometer, which represented a receiver with an input signal sensitivity of $0.5 \mu \mathrm{V}$ and a stepped tuning in the $0.1-10.0 \mathrm{MHz}$ frequency range (Izhovkina et al., 2009). The frequency tuning step was $25,50,100 \mathrm{kHz}$, the bandwidth at a receiver input was $15 \mathrm{kHz}$, and the dynamic range of input signal level variations was $80 \mathrm{~dB}$. An electric dipole antenna 


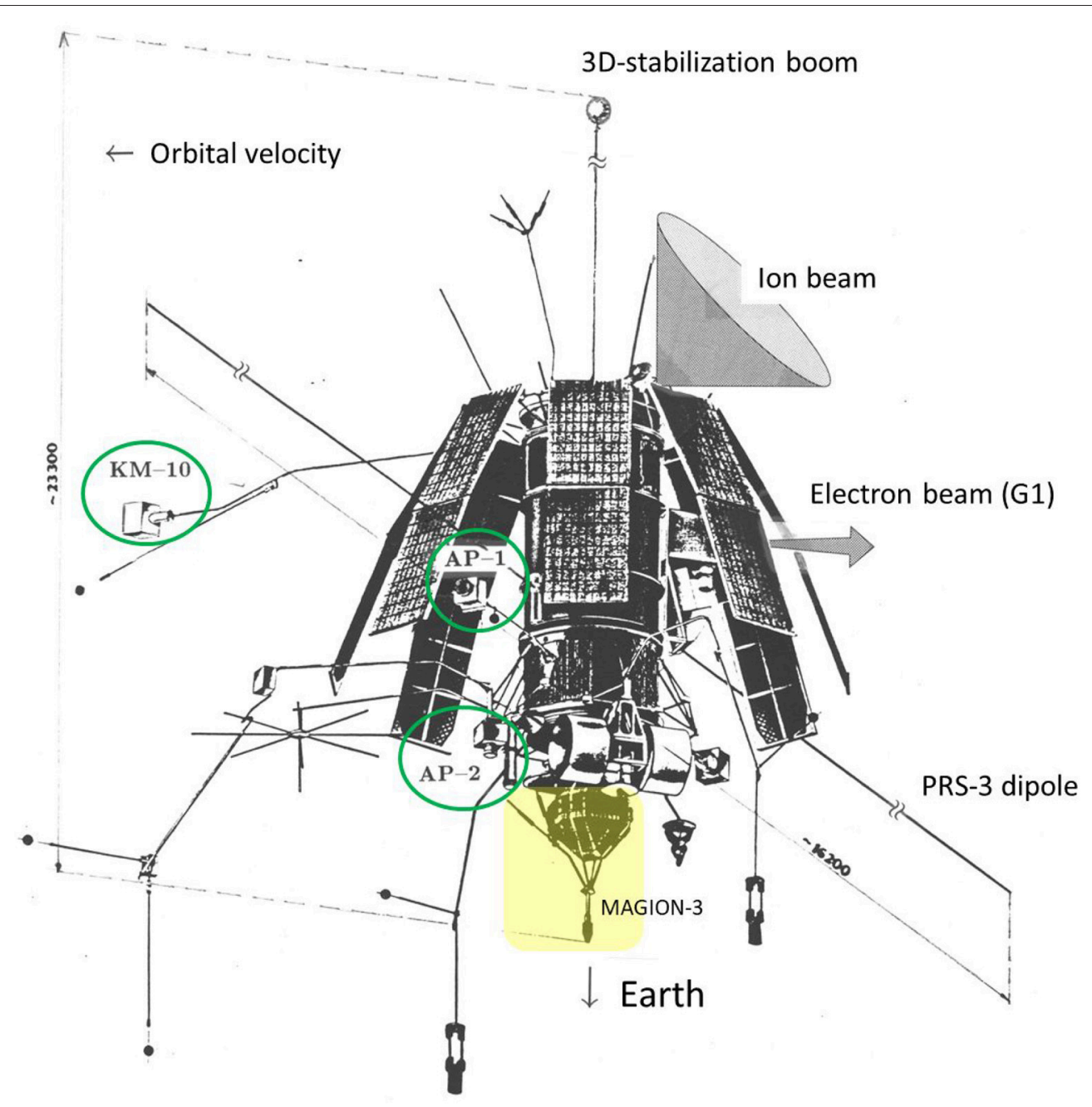

FIGURE 1 | IK-25 deployed (before MAGION-3 separation, its launch position highlighted in yellow). Positions of the KM-10, AP-1, and AP-2 particle sensors, the PRS-3 dipole antenna, and the electron/ion injection directions are indicated. (Adapted from Prech, 1995).

of the VCH-VK complex with a total length of $15 \mathrm{~m}$, parallel to the Earth's surface, was used as a device sensor (Figure 1).

The low-frequency wave instrument NVK-ONCH registered magnetic field spectrum in the range 8-969 Hz and amplitudes at fixed frequencies 9.6 and $15.0 \mathrm{kHz}$ (Oraevsky et al., 2001). Measurements of the sensitive flux-gate magnetometer SGR-5 and the spacecraft service magnetometer were used to assess a level of magnetic field fluctuations at lowest frequencies. Quasi-steady electric field $0.1-10 \mathrm{~Hz}$ components and VLF electric field spectra were measured by a system of double electric probes connected to inputs of the DEP-2 instrument and the NVK-ONCH wave complex (Baranets et al., 2007).

\subsubsection{MAGION-3 Short Description}

The size of the object was $0.85 \times 0.60 \mathrm{~m}$ diameter $(2 \mathrm{~m}$ with deployed booms), its weight was $52 \mathrm{~kg}$. The orbital speed could be corrected using pressurized neutral gas (nozzles parallel/antiparallel with the satellite axis). The satellite axis was oriented approximately along the local geomagnetic field (builtin permanent magnet $30 \mathrm{Am}^{2}$ with week dumping) (Triska et al., 1990).

The suprathermal particle spectrometer MPS/PPS was a simplified version of the PEAS instrument (Nemecek et al., 1994). It measured energy distribution of electron and ions in one halfplane containing the satellite main axis, divided to 6 angular sectors (SEA-A and SEA-B toroidal-cut analyzers, $25^{\circ}$ width). The system was complemented by two narrow field-of-view electron energy analyzers MP-A and MP-B (parallel/antiparallel to the satellite axis, respectively) to obtain a full-range electron pitch-angle distribution (8 channels with $30^{\circ}$ spacing).

The energetic particle sensor DOK-A-S registered energetic electron and proton fluxes parallel and perpendicular to the satellite axis using two pairs of silicon detectors (Prech et al., 2002; Baranets et al., 2007). 


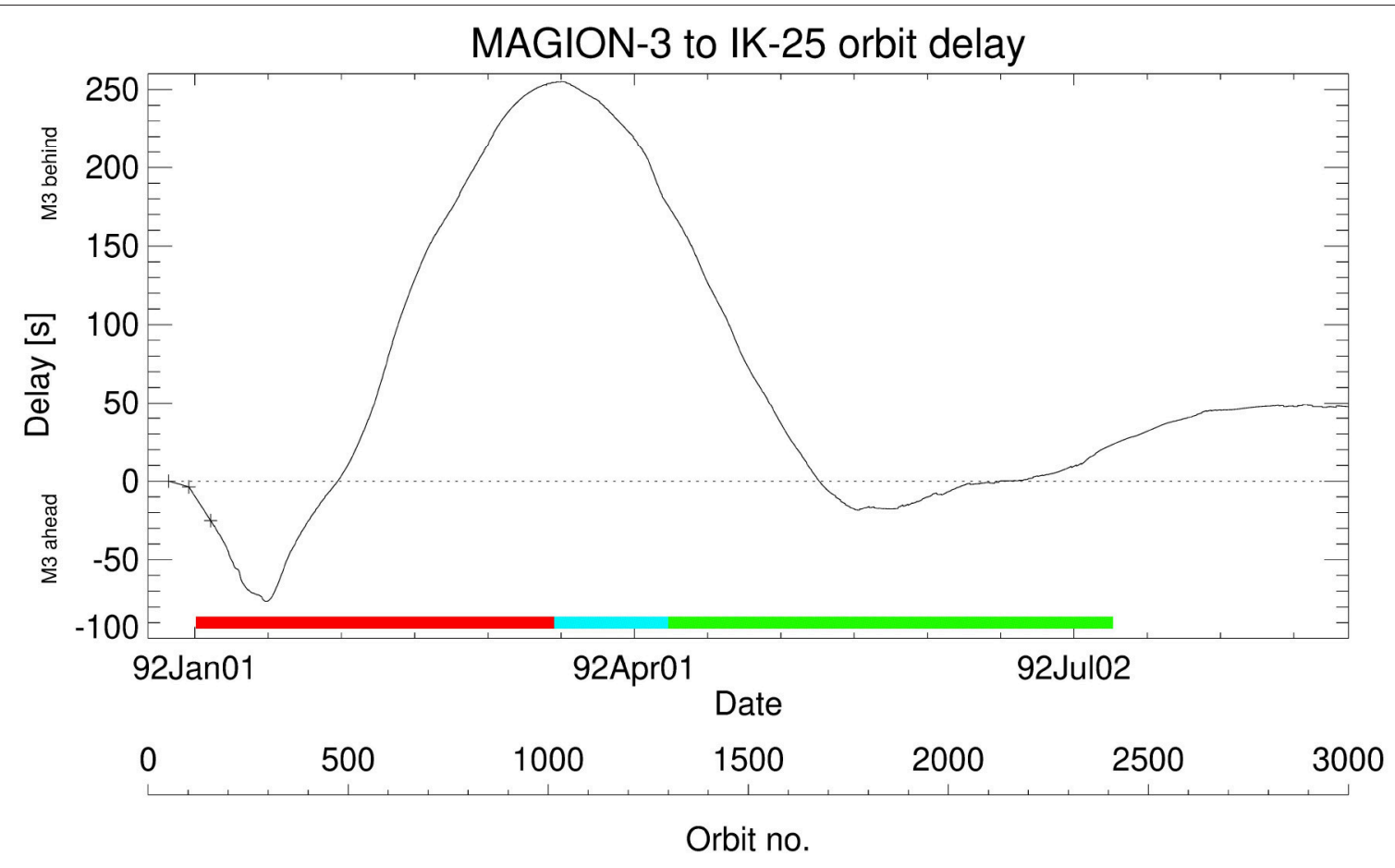

FIGURE 2 | MAGION-3 to IK-25 in-orbit delay. The delay absolute minimum corresponds to $\sim 580 \mathrm{~km}$ distance ahead, the absolute maximum to $\sim 1$, $900 \mathrm{~km}$ distance behind. The red bar denotes the season where both UEM and UPM injections were performed, later only the experiments with the UPM injector were done-blue with xenon plasma, green - neutral xenon releases only.

The wave experiment provided spectra of two electric and one magnetic field components in ELF/VLF ranges (Triska et al., 1990; Baranets et al., 2007). Waveforms of selected electric/magnetic components up to $60 \mathrm{kHz}(2-3 \times 8 \mathrm{~Hz}-20 \mathrm{kHz}$ or narrow-band) could be directly transmitted to ground. The PRS-2-S radio-spectrometer provided spectra with $\Delta f 15$ or 50 $\mathrm{kHz}$ resolution in the $\mathrm{HF}$ frequency range $0.1-10 \mathrm{MHz}$ (one electric field component, $3 \mathrm{~m}$ length dipole) (Rothkaehl and Klos, 1996).

\subsection{Active Experiment Methodology}

The MAGION-3 was separated on December 28, 1991 from the main satellite and drifted away with a speed $\sim 5 \mathrm{~km} /$ day. Several tens of orbit corrections were made to keep the mother-daughter relative distance within range -580 to $+1,900 \mathrm{~km}$. The in-orbit MAGION-3 to IK-25 delay evolution is depicted in Figure 2. Color bars in this figure mark different campaigns of active experiments, planning of which was affected by the electron and ion injectors working status. About 200 active experiments with electron and plasma injections in various configurations were performed till July 1992 within the satellite relative distance 70-500 km. Planned experiments in near (> $10 \mathrm{~m}$ to $0.4 \mathrm{~km}$ ) and mid-range $(\sim 1-10 \mathrm{~km})$ zones could not be performed for technical reasons. Two-point passive measurements at distances $100-2,000 \mathrm{~km}$ continued till the MAGION-3 end-of-life in August 1992. Ionospheric investigations by IK-25 lasted till July 1993.
The IK-25 satellite was controlled from Soviet telemetry stations and selected scientific data were delivered to ground via its primary telemetry channel (RTS), mostly from its onboard telemetry memory with downloads once per day but real-time telemetry sessions were also started frequently in the regions of satellite visibility to allow better data time resolution. Except the active experiment devices and diagnostic equipment IK-25 also included a complementary telemetry system STO-AP developed by teams from Hungary, former USSR, Poland, and Czech Republic. The system STO-AP provided an interface for the scientific instruments, management of operation modes, preliminary processing of the scientific information, independent control of onboard experiments, formation of the TM frames and transfer of the telemetry information to the ground-based stations at Czech Republic (Panska Ves), Russia (Troitsk, Apatity, Tarusa), and Germany. STO-AP enabled higher volume/better time resolution of science data mostly during real-time telemetry sessions lasting about 10 min (memory replay sessions with limited data volume were also sporadically performed). Drawback of this approach was the IK-25 visibility regions from the RTS and STO-AP reception stations did not fully overlap (resulting many active experiments controlled via RTS were not fully covered by scientific data routed via STO-AP). Beside it, the STO-AP telemetry channel (137/400 $\mathrm{MHz}$ bands) was often affected during strong xenon plasma injections (short drop-outs and wide data gaps were present during such sessions). The limited total telemetry capacity 


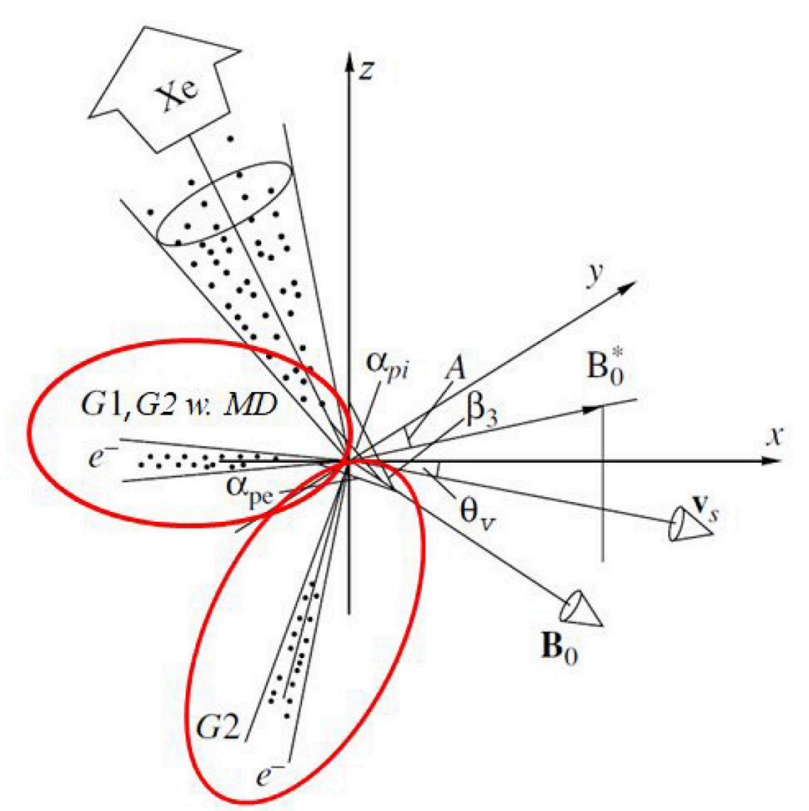

FIGURE 3 | Injection directions, orientation angles of the magnetic field $\mathrm{B}_{0}$ and velocity $v_{S}$ in the satellite frame of reference $x y z$; the $z$ axis is directed from the Earth. "Xe"-UPM xenon plasma/neutral gas release cone, G1 (G2 with the magnetic deflector MD) and G2 (without the deflector) denote possible electron gun UEM injections. $\alpha_{p i}, \alpha_{p e}$ mark the ion and electron injection pitch angles. (Reprinted from Baranets et al., 1999, with permission from Elsevier).

necessitated trade-offs in the scientific instruments operational modes and their resolution, during some orbits not a complete parameter set could be studied.

The MAGION-3 daughter satellite was controlled and scientific data transmitted via the STO telemetry to the Panska Ves observatory (Czech Republic) mostly during real-time sessions as its onboard data memory volume was limited (4 $\mathrm{MB})$. For this reason the satellite pair visibility also affected the simultaneous data coverage of active experiments as performed from the main IK-25 satellite, especially during periods of larger spacecraft separation.

The Panska Ves visibility requirement made the altitude, invariant latitude (INL)/L-shell, magnetic local time (MLT) parameters of the APEX active experiments bound. As the active experiments were run above the north hemisphere, downward electron injections during day were toward the north magnetic pole (near mirror point in dense ionoshere below) and upward electron injections (during night) were directed toward the south magnetic pole (distant mirror point behind the rarefied top ionoshere/magnetosphere). This brings difficulties to separate some physical parameter dependencies. The electron and plasma injectors onboard IK-25 had many working/modulation modes and the elliptical orbit of the satellite pair allowed performing the active experiments for different ionospheric parameters. Ideally, a certain injection configuration should be repeated several times under different ambient conditions, but in reality only one usable dataset was captured for many configurations.
Despite these technical difficulties a rich database of observational data from active experiments of the APEX project was collected and many interesting scientific results were published.

\section{RESULT OVERVIEW}

\subsection{Spacecraft Charging and Neutralization During Active Emissions}

Beside the complex IK-25 design geometry the evaluation of the spacecraft $(\mathrm{s} / \mathrm{c})$ charging properties was complicated also by other factors. The IK-25 body was painted and its surface was assumed equipotential but the conductivity was not characterized in publicly available data. The scientific payload was painted or covered by multi-layer insulation (MLI) and connected to the spacecraft ground and the surface again assumed equipotential (not guaranteed). Solar panel surfaces probably were not equipotential-the positive potential end was probably exposed to the ionospheric plasma (dayside effects on the satellite potential). Also, after the end of all injections the spacecraft potential return to its "quiet" level immediately during night, but with a time constant of few minutes during day. A full charge balance analysis has also to consider the stabilization boom/weight at $\sim 20 \mathrm{~m}$ distance. The electrical current across the boom was not monitored, unfortunately.

The KM-10 sensor box (placed ahead of the spacecraft in the ram direction) was kept floating near the ionospheric plasma potential $\left(\delta \Phi \sim-0.2\right.$ to $-0.7 \mathrm{~V}$ for $T_{e} \sim 0.1-0.3 \mathrm{eV}$, not considering photocurrent/suprathermal electrons). The KM-10 floating probe potential $\Phi_{p}$ (normally positive against s/c) was widely used as a spacecraft potential proxy $\left(\Phi_{s} \approx-\Phi_{p}+\delta \Phi\right)$, but its design range $\pm 90 \mathrm{~V}$ is doubtful ( -10 to $+16 \mathrm{~V}$ limitation was observed in practice). The KM-10 data time resolution $(0.2 s)$ and UEM/UPM injection data $(0.32 / 0.08 s$ resolution) unfortunately do not allow to study transient charging phenomena during the injections on/off or detail profiles for individual sub-millisecond electron injection pulses. As the Debye length near the apogee $\left(\sim 10 \mathrm{~V}, 10^{4} \mathrm{~cm}^{-3}\right)$ is approximately $1 \mathrm{~m}$, the KM-10 might not be always out of the spacecraft Debye sheath. Also, this spacecraft potential proxy is considered unreliable when the KM-10 ion density $N_{i}$ drops to its lower limit $\left(\sim 10^{8} \mathrm{~m}^{-3}\right)$. The parameter reliability was discussed in more details in Prech (1995).

During the active experiments with electron injections, the secondary electron emission from the spacecraft surface due to hot electron collection (return currents) probably also affected the spacecraft charge balance. Up today no detail and realistic IK-25 charging model is publicly available. A simplified model is discussed by Zilavy et al. (2003).

\subsubsection{IK-25 Potential Outside of Active Experiments}

Prech et al. (1999) investigated the "quiet time" behavior of the IK-25 spacecraft properties. Figure 5A depicts a potential difference between the KM-10 floating probe and the satellite $\left(\Phi_{p}\right)$ as a function of the altitude. The data were collected during intervals preceding the electron/plasma injections and the satellite potential can change for several volts depending on the part of the orbit (with changing the altitude, ambient plasma 


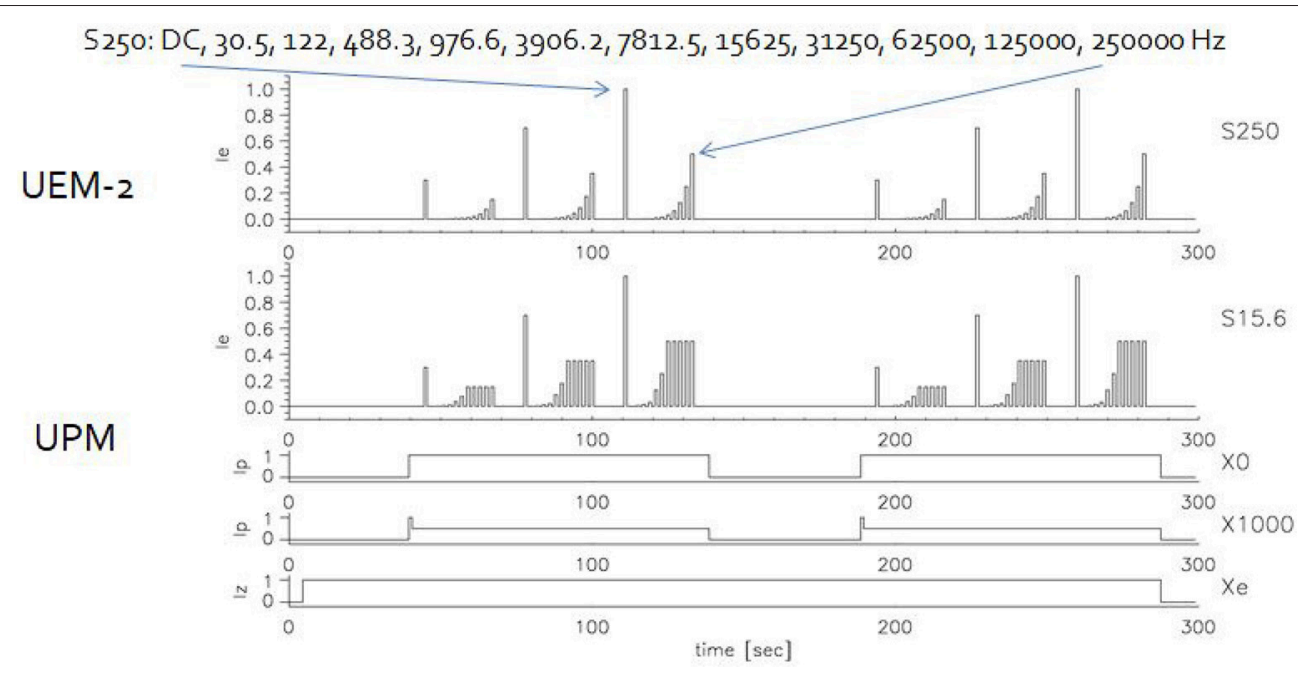

FIGURE 4 | Mean injection current profiles for different beam modulation modes of the UEM-2 and UPM injectors. Top two panels-UEM-2 modes S250 and S15.6; bottom three panels - UPM DC mode F0, modulated mode F1000, and neutral xenon gas release. (Adapted from Prech, 1995).

density, and electron temperature), the satellite always charges negatively. The dayside/nightside orbits split the observations to two branches. The night branch (the satellite was in the Earth's shadow) is a rising function of the altitude which was explained with the electron temperature also rising with the altitude. Figure 5B shows the same data as a function of the ion density $N_{i}$ measured by the KM-10 device. The higher potentials (more negative s/c charging $\Phi_{s}$ ) on the dayside branch are assumed due to the solar panels connected through a small resistance to the ambient plasma at low altitudes. This resistance is a function of density and the effect gradually disappears at altitudes above $2,500 \mathrm{~km}$ or densities below $10^{9} \mathrm{~m}^{-3}$.

\subsubsection{IK-25 Potential During the Electron Beam Injections With Xenon Plasma Neutralization}

The IK-25 spacecraft charging during the electron beam injections with the xenon plasma neutralization can be summarized to following conclusions (Prech, 1995, 2002; Prech et al., 1995, 1999; Nemecek et al., 1997):

- For ambient plasma densities $N_{i}>10^{11} \mathrm{~m}^{-3}$ the electron beam charge was well neutralized, $\Phi_{s}<15 \mathrm{~V}$ for S250 and F40K modes peak currents, KM-10 density $N_{i}$ measured ahead of the spacecraft was not further increased during the xenon injections.

- For ambient plasma densities $N_{i}<10^{11} m^{-3}$ and the xenon plasma release during day kept the KM-10 ram density $N_{i} \sim 8 \times 10^{10} \mathrm{~m}^{-3}$ and the electron beam charge was wellneutralized, the neutralization appeared even better during night, the KM-10 ram density $N_{i} \sim 1.5 \times 10^{11} \mathrm{~m}^{-3}$ and the spacecraft potential relaxed to its "quiet" level (for example see Figure 6B).

- For lower ambient plasma densities during neutral xenon gas / hollow cathode releases (Xe-A/Xe-B) the spacecraft is charged to more than $+20 V$ against its "quite" level.
The KM-10 floating potential reached a negative saturation or after shortly negative excursion it traveled to positive saturation and as such it could not be used as a reliable IK-25 charging monitor (Figure 6B). As accelerated cold ionospheric electrons were not registered by the PEAS spectrometer, the spacecraft potential was within limits $\sim 20 \mathrm{~V}<\Phi_{s}<\sim 50 \mathrm{~V}$.

- During the modulated electron beam injection (S250), when $\Phi_{p}$ was not in saturation, $\sim 1 s$ positive pulses in the spacecraft potential were observed (rising/falling edges $<0.2 s$ ) - see e.g., Figure 6A. Their amplitude increased with the mean electron pulse current level until it reached the unmodulated (F40K) levels. The amplitude was larger during Xe-B mode than with the main xenon plasma injection showing worse electron beam neutralization in this mode.

\subsubsection{IK-25 Potential During the Xenon Plasma Injections}

The IK-25 spacecraft potential behavior during the UPM neutral gas releases and xenon plasma injections (UEM not active) was studied by Prech et al. (1999), further examples and discussion are contained in Prech $(1995,2002)$. It was found that the release of the neutral xenon alone did not affect the spacecraft potential under conditions of the APEX experiment. The change of the spacecraft potential $d F P$ induced by the release of the xenon plasma was up to $\sim \pm 10 \mathrm{~V}$ (e.g., Figures 7A,B). Such a change was considered reasonable, as the temperature of the plasma in the injector output was about $50 \mathrm{eV}$, sharply decreasing with the distance from the spacecraft due to adiabatic expansion of the xenon plasma cloud. A dense conducting cloud (plasma contactor) created by the released plasma behind the spacecraft was expected to effectively collect electrons from the ambient plasma. Being partly in the spacecraft wake the cloud contribution to the ion current is found smaller. The dimension of this cloud was expected comparable with the dimensions of the "diamagnetic 

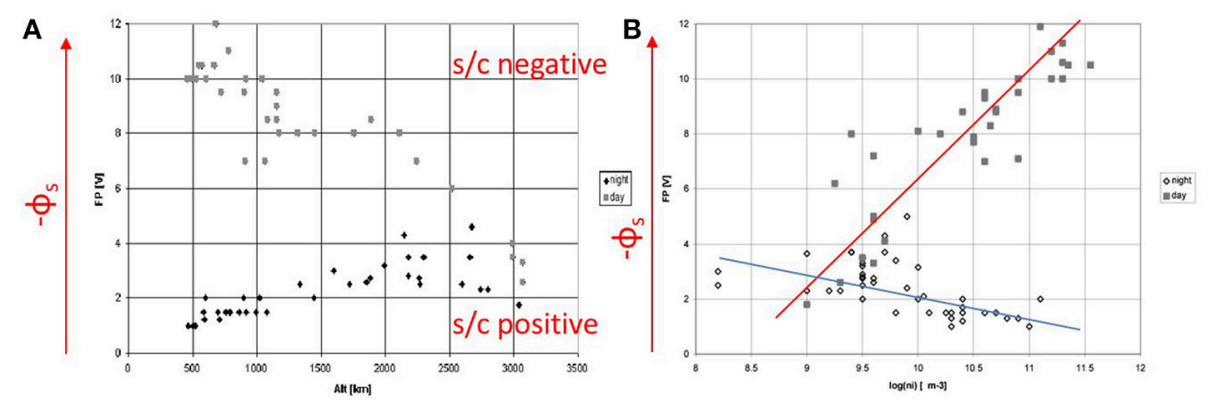

FIGURE 5 | IK-25 spacecraft potential outside of active experiment intervals. The plots show the potential difference between the KM-10 floating probe and the spacecraft body as a function of the altitude (A) and the ambient plasma density (B). The spacecraft is charged negatively for positive KM-10 floating potentials. (Reprinted from Prech et al., 1999, with permission from Elsevier).

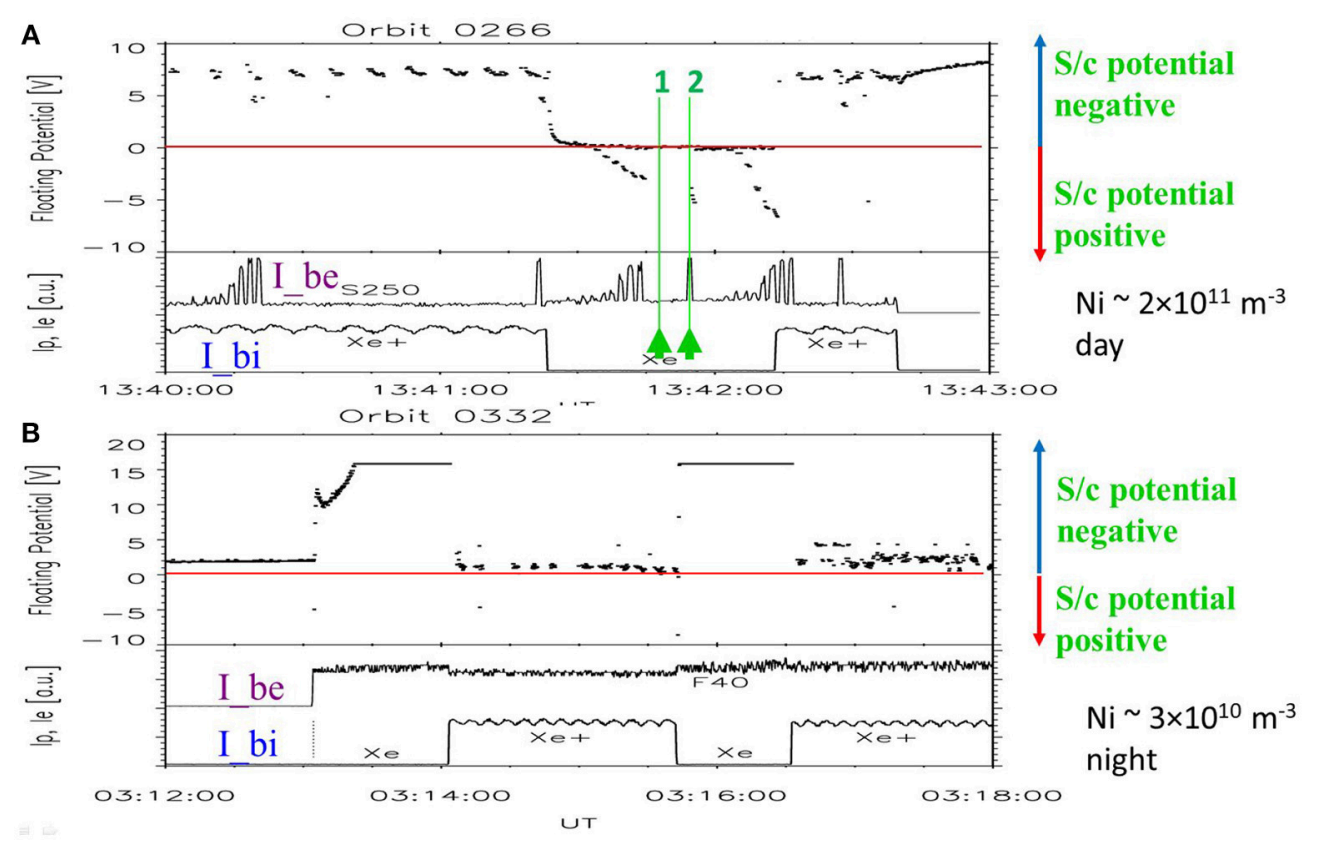

FIGURE 6 | Examples of the IK-25 spacecraft potential behavior during electron beam injections. The plots show the KM-10 floating probe potential, mean UEM electron beam and UPM plasma injector currents: (A) Orbit 0266-dayside modulated electron beam (S250 mode, G1 injector) with xenon gas/plasma neutralization (FO mode), green vertical lines assigned 1 and 2 mark instants with the UEM beam base current and UEM beam maximum current (DC pulse); (B) Orbit 0332 - nightside electron beam injection (F40K mode, G2 injector with magnetic deflector) with xenon gas/plasma neutralization (F0 mode). (Reprinted from Prech et al., 1995, with permission from Elsevier).

phase" of the plasma cloud expansion proposed by Hausler et al. (1986).

The electron current collected by the cloud should rise linearly with ambient plasma density, but this rising current causes an increase in the negative spacecraft potential. The result was the observed linear relationship between $\log N_{i}$ and $d F P$ (Figure 7C). The collected electron current as the difference between those electrons actually collected and those leaving the plasma cloud was found either positive or negative. If the number of electrons collected from the ambient plasma is higher than the number leaving the cloud then the spacecraft potential change vs. "quiet" level $d F P$ is negative, otherwise it is positive. Altitude, ambient plasma temperature, and day/night dependencies were found small for this effect.

The DC xenon plasma emission was studied in a simple model of a planar floating probe, spherical satellite, and plasma cloud by Zilavy et al. (2003). The authors stress the importance of suprathermal electron tail and ion mass composition (affecting the $\mathrm{H}^{+}, \mathrm{O}^{+}$ion ram energy) of ionospheric plasma for charge balance. The model can explain the spacecraft potential change of both polarities suggesting the ionospheric $\mathrm{O}^{+}$ions are less effectively collected by the plasma cloud due to their higher ram kinetic energy. 

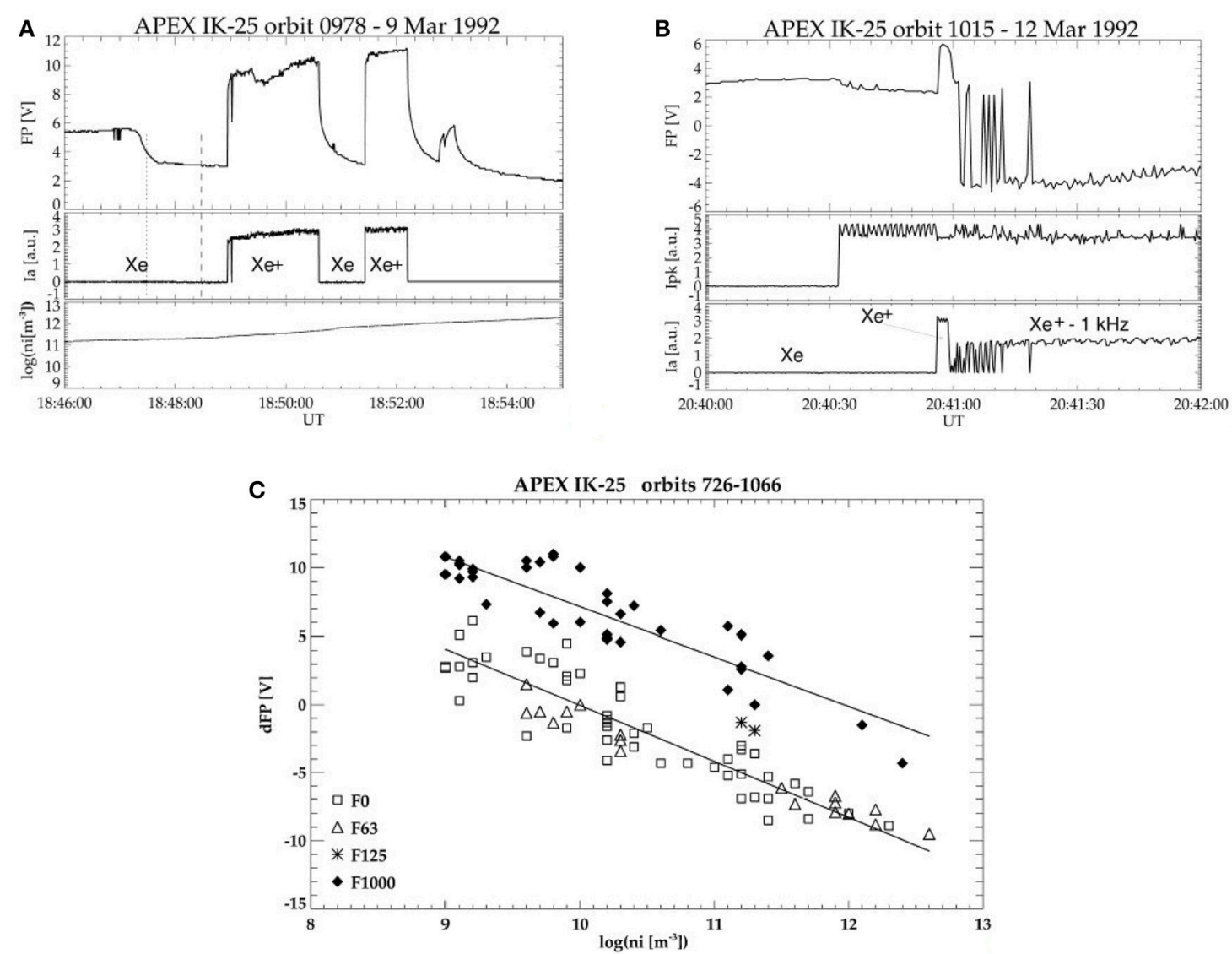

FIGURE 7 | Examples of the IK-25 spacecraft potential behavior during the xenon plasma injections. (A) (Night DC injection F0 mode at $\sim 440 \mathrm{~km}$ altitude) shows the KM-10 floating probe potential, the mean UPM ion current, and the ambient plasma density (KM-10). (B) (Dayside DC/1 kHz injection F1000 mode at $\sim 2,900 \mathrm{~km}$ altitude) depicts the KM-10 floating probe potential, the UPM hollow cathode current, and the mean UPM ion current (in both panels Xe stands for the neutral xenon release intervals, $\mathrm{Xe}+$ for the xenon plasma injections). (C) Change of the spacecraft potential during the xenon plasma releases as a function of the ambient plasma density for different UPM modulation modes. (Reprinted from Prech et al., 1999, with permission from Elsevier).

\subsection{Neutral Xenon Release Experiments}

During the xenon plasma injection experiments of the APEX programme, about 20 experiments were performed when the UPM device worked uniquely in the mode of neutral xenon gas release. Additional experiments included simultaneous injections of electron beam and neutral xenon. Under conditions of the experiment the kinetic energy of the xenon atoms motion relative to the ionospheric plasma $(\sim 25-40 \mathrm{eV})$ exceeded the xenon first ionization potential $(\sim 12.1 \mathrm{eV})$, so the necessary condition of the anomalous ionization was satisfied. The neutral xenon releases occurred over the full altitude range 450-3,000 $\mathrm{km}$ and variety of experimental conditions (geomagnetic field strength, ambient plasma density, injection angle vs. magnetic field direction, illumination etc.) allowing comparison with other experiments.

Oraevskii et al. (1999) and Choueiri et al. (2001) analyzed the influence of the neutral xenon releases to spectra of highfrequency plasma turbulence, accelerated electron spectra, and spectra of electric field fluctuations near lower-hybrid resonance. They also studied dependence on angle between the magnetic field and the gas injection direction. The experimental data show the ambient plasma response to the neutral gas injection including electron temperature and anisotropy increase and amplification of wave activity practically over full range of registered frequencies. Theoretical considerations of these papers are consistent with the observed data. Although only small flows of neutral xenon gas $(\sim 3 \mathrm{mg} / \mathrm{s}$ ) were released during several minute lasting intervals, due to its collisional interaction with the background ionospheric plasma a sufficient number of "seed" energetic ions was created so as the CIV-related electron heating could be observed. Newly created xenon ions by charge exchange collisions, electron impact ionization, photoionization, and scattering of ionospheric plasma ions were considered in their analyses. The calculated ionization yield smaller than $1 \%$ corresponds to not observable changes of plasma density. Ion-ion lower-hybrid wave instability was expected to reach distances 3-100 $\mathrm{m}$. In the spacecraft reference system these waves have a perpedicular to the magnetic field phase velocity component in the range of reflected ion speeds $0-7$ $\mathrm{km} / \mathrm{s}$, but their group velocity is directed almost parallel to the 
magnetic field. For this reason the waves can reach the spacecraft and be detected. Unfortunately, the proper frequency band diagnostic was not operating, but simultaneous narrow- and wide-band $\mathrm{HF}$ emissions in the range 3-10 $\mathrm{MHz}$ during dayside xenon gas emissions with pitch angles $85-115^{\circ}$ were observed. Intensification of HF wave activity was not observed during nightside emissions (pitch angles $57-71^{\circ}$ ). The wave frequency bands were flat and did not evolve along the orbit (magnetic field strength). The authors think that the observed waves could be a symptom of turbulent fluctuations connected with the instability, but without a theoretical explanation. According to the authors, it is possible that the presence of the solar flux and not the emission pitch angle is the controlling parameter for the observed effects. In such a case, the role of the solar flux in affecting HF wideband activity through intermediary effects such as plasma enhancement due to photoionization may be worthy of investigation.

\subsection{Beam-Plasma Interaction}

\subsubsection{Hot Electron Pitch-Angle Distributions During Electron Beam Injections}

Plasma environment in the vicinity of the IK-25 spacecraft and return currents during the UEM electron beam injections were studied in Prech (1995), Nemecek et al. (1997), and Prech et al. (1998) using the PEAS electron and ion spectra with following conclusions:

- Hot electrons as a part of the return current were registered by both the AP-1 and AP-2 sensors with energies peaking between $\sim 10^{2}$ and $10^{3} \mathrm{eV}$, electrons were preferentially accelerated perpendicular to the magnetic field. The acceleration mechanism was weaker during simultaneous xenon plasma release.

- The hot electron flux and energy increased with the mean primary beam current, but the PEAS time resolution did not allow to observe any resonance effects depending on the electron beam modulation frequency.

- The energy/pitch-angle distributions allowed to distinguish several electron populations attributed to accelerated ambient plasma electrons (at lower energies) and scattered beam electrons (few $k e V$ energies). No electrons with energies accelerated above the primary beam energy were found in the PEAS spectra.

- Hot suprathermal electrons constituting a part of the return currents arrived with comparable fluxes from directions parallel and antiparallel to the magnetic field, i.e., not only from the area behind the spacecraft disturbed by the injected beam but also from the ahead region that could be affected only by the return currents and secondary products of the beam-plasma interaction.

- The energy and flux of these electrons decreased for pitch angles closer to parallel/antiparallel directions indicating the space localization of the acceleration process.

- The electron distributions were not homogenous in spacethe $\mathrm{AP}-1$ sensor registered distributions narrower in energy and lower fluxes than the AP-2 sensor mounted close to the spacecraft body.
- The AP-2 electron spectra showed differences in pitch-angle spectra for sectors looking inside (group 1) and outside (group 2) of a cylinder shell containing the primary beam electrons (an example is presented in Figure 8). The asymmetry of the electron fluxes could be explained with $E \times B$ drift but the corresponding local electric fields would reach $\approx 100 \mathrm{~V} / \mathrm{m}$.

- The observations supported the picture where the primary beam created a hollow cylinder with its shell full of hot electrons, the cylinder extended both parallel/antiparallel to the magnetic field. The AP-2 sensor was located right in the hot shell. The physics of such beam expansion is, of course, complex and as such studied theoretically including computer simulations and experimentally both in laboratories and in space. One of the relatively recent computer simulations related to the APEX experiment was made by Lizunov et al. (2002). The beam current profile and generally the beamplasma interaction in context of the APEX experiments was recently investigated e.g., by Baranets et al. $(2012,2017)$.

No significant ion fluxes were registered by the PEAS sensors during the UEM and UPM active injections (in different mode combinations), the counts usually remained near the noise level (Nemecek et al., 1997). Only sporadically an ion group with energy corresponding to the UPM acceleration voltage was registered in a narrow spatial angle-usually just one sector. Although the PEAS sensors had no mass resolution capability it was assumed these were ions from the plasma cloud edge that after one gyro-revolution returned to the PEAS input aperture.

More recently, Budko et al. (2003) also analyzed the PEAS electron and ion distribution behavior during the electron beam injections related to the HF wave emissions (see section 3.3.3). They emphasized detection of very short-term bursts of accelerated ions with energies up to several hundreds of electronvolts immediately after switching on the electron gun. The effect again corresponds to generation of strong electric fields in the near zone.

\subsubsection{Plasma Environment in the IK-25 Spacecraft Vicinity, Diamagnetic Cavity}

The UPM plasma cloud density evolution in the APEX active experiments were estimated by Volokitin et al. (2000). The kinetic energy $200 \mathrm{eV}$ of accelerated xenon ions corresponds to $\sim 17 \mathrm{~km} / \mathrm{s}$ speed. For the typical UPM emission parameters $2.5 \times 10^{19}$ ions/s $(\sim 3 \mathrm{mg} / \mathrm{s})$ assuming density decreased as $\sim$ $1 / R^{2}$ the xenon plasma expanded to the ambient plasma density levels $\sim 10^{10} \mathrm{~m}^{-3}$ at distances $R \simeq 300-400 \mathrm{~m}$. From similar estimations the xenon plasma dynamic pressure exceeded the geomagnetic field pressure for $R[m]<2.5 \times 10^{5} / B_{0}[n T]$. For the APEX orbit $\left(B \sim 3-5 \times 10^{4} n T\right)$ the plasma cloud could expel the geomagnetic field and create a diamagnetic cavity to distances 5-10 $m$ from the spacecraft (the xenon ion gyroradius $1-2 \mathrm{~km}$ ) due to electrical polarization of plasma cloud. While the injected energetic xenon ions move more-or-less freely across the magnetic field, the electrons are magnetized and follow the magnetic field lines. The electrons partly drift due to the polarization electric field across the magnetic field and at the same time they drag the magnetic field lines in their effort to 


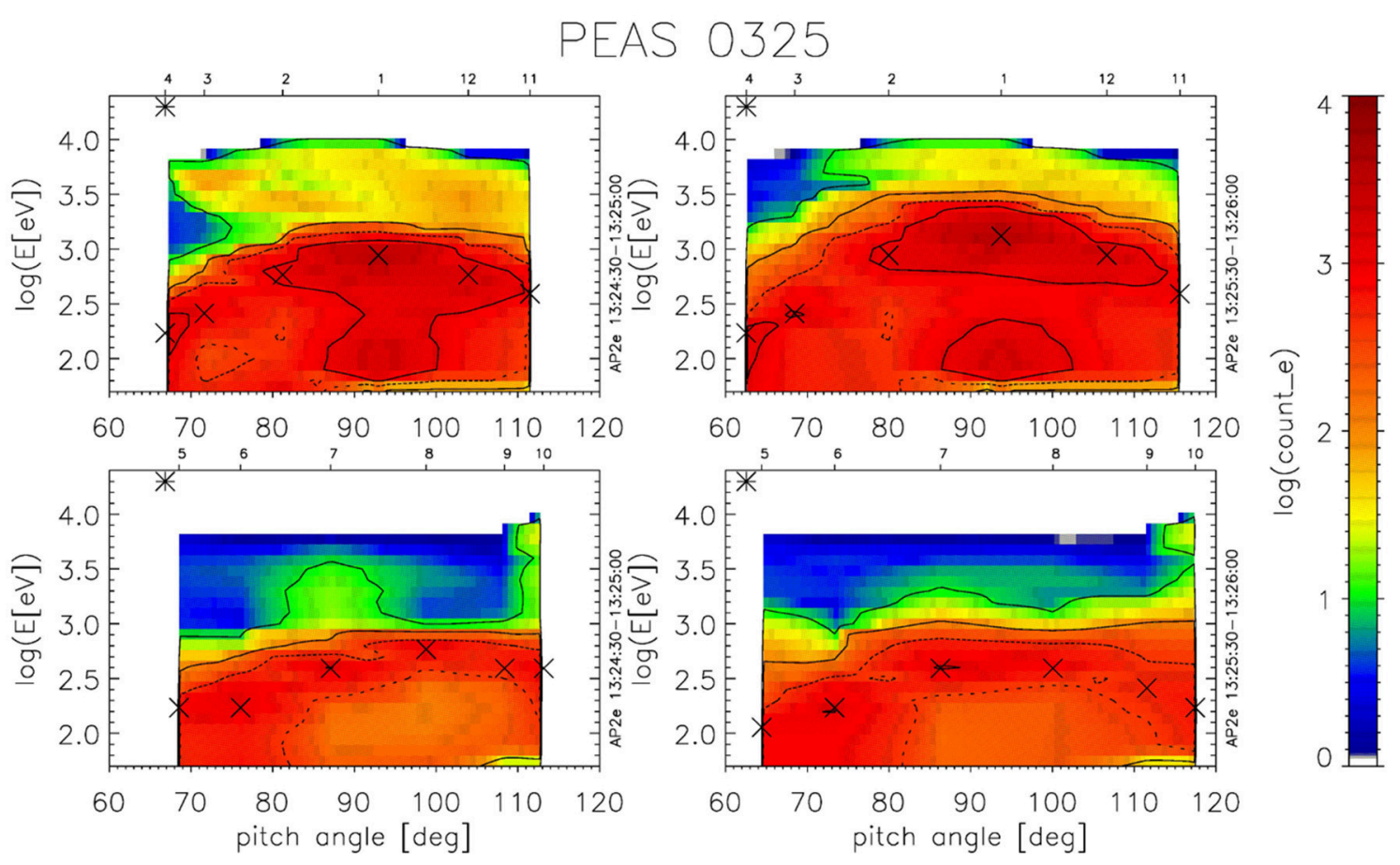

FIGURE 8 | The electron energy and pitch angle distribution obtained with the PEAS AP-2 sensor during electron beam emission (orbit 325 , G2 injector with magnetic deflection, F40K mode) accompanied by xenon plasma injection (FO mode) on the left and neutral xenon (Xe-B) on the right. (Top) AP-2 sectors seeing the electrons from inside the beam flux tube (group 1), (Bottom) Sectors seeing the electrons from outside (group 2). The UEM injection pitch angle denoted by *. (Reprinted from Nemecek et al., 1997, copyright 1997 by the American Geophysical Union).

follow the cloud ions. However, this process could be noticeably depressed if the ambient plasma electron density is sufficient to support the parallel electric current neutralizing the polarization electric field. Volokitin et al. (2000) studied the diamagnetic cavity formation during the IK-25 xenon plasma injection using two magnetometers (see Figure 9). The SGR-5 magnetometer mounted on a boom at least 7-12 $\mathrm{m}$ from the xenon plasma beam registered magnetic field fluctuations with amplitude $\sim$ $10 n T$ while the service magnetometer mounted very near the injected cloud observed magnetic depressions 1,500-2,000 $n T$. The experimental data documented the active extrusion of the geomagnetic field in the initial phase of the plasma cloud expansion. The authors compared the observational results with a theoretical model based on ideas from analysis of previous experiments PORCUPINE (Hausler et al., 1986; Oraevsky et al., 2003) and AMPTE (Mishin et al., 1988).

\subsubsection{Beam-Plasma Interaction, ELF/VLF, and HF Waves Generation}

Near-zone VLF wave observations (NVK-ONCH instrument) during the APEX xenon plasma injection have been studied by Mikhailov et al. (1998, 2000). While the unmodulated xenon plasma emission was accompanied by a broadband VLF noise, during the $1 \mathrm{kHz}$ and $125 \mathrm{~Hz}$ beam current modulation (F1000 and F125 modulation modes), the basic frequency and its harmonics were registered in the magnetic field data. Simultaneous disturbances in electron temperature and plasma density from the KM-10 device were detected. While the onset of fast magnetic field variations coincided with the plasma injection switch-on, they lasted much longer after the injection off, with time-varying spectral amplitude profiles across the ELF/VLF range. The authors attributed the observed delays to a joint movement of the satellite and a packet of waves created inside the plasma cloud (assuming different wave modes were excited during the injections and some of them were able to travel in the direction of the satellite velocity according to the authors). Similar results were obtained also in previous higher flow, ram directed, xenon neutral gas releases from the ACTIVE Interkosmos-24 satellite (Klos et al., 1998).

Kiraga et al. (1995, 1998) analyzed HF emissions during modulated electron beam injections (F40K mode, $2 \mu$ s pulses, neutral xenon simultaneous release) in the middle latitude dayside ionosphere (altitude $\sim 700-1,100 \mathrm{~km}$ ) and near the nightside perigee (altitude $\sim 415-470 \mathrm{~km}$, middle and high latitudes). In the regime of strong beam-plasma interaction, the HF dipole antenna was effectively screened from the HF broadcasting stations radiation as probably a large volume of excited plasma around the emitting satellite scattered these waves. Complex structures of peaks near harmonics of local plasma, cyclotron, and upper-hybrid frequencies were present in the HF wave spectra of PRS-3 during the electron injections, varying with ionospheric parameters and evolving injection configuration along the orbit. Dublet of local plasma and upper hybrid frequency peaks was the most prominent 

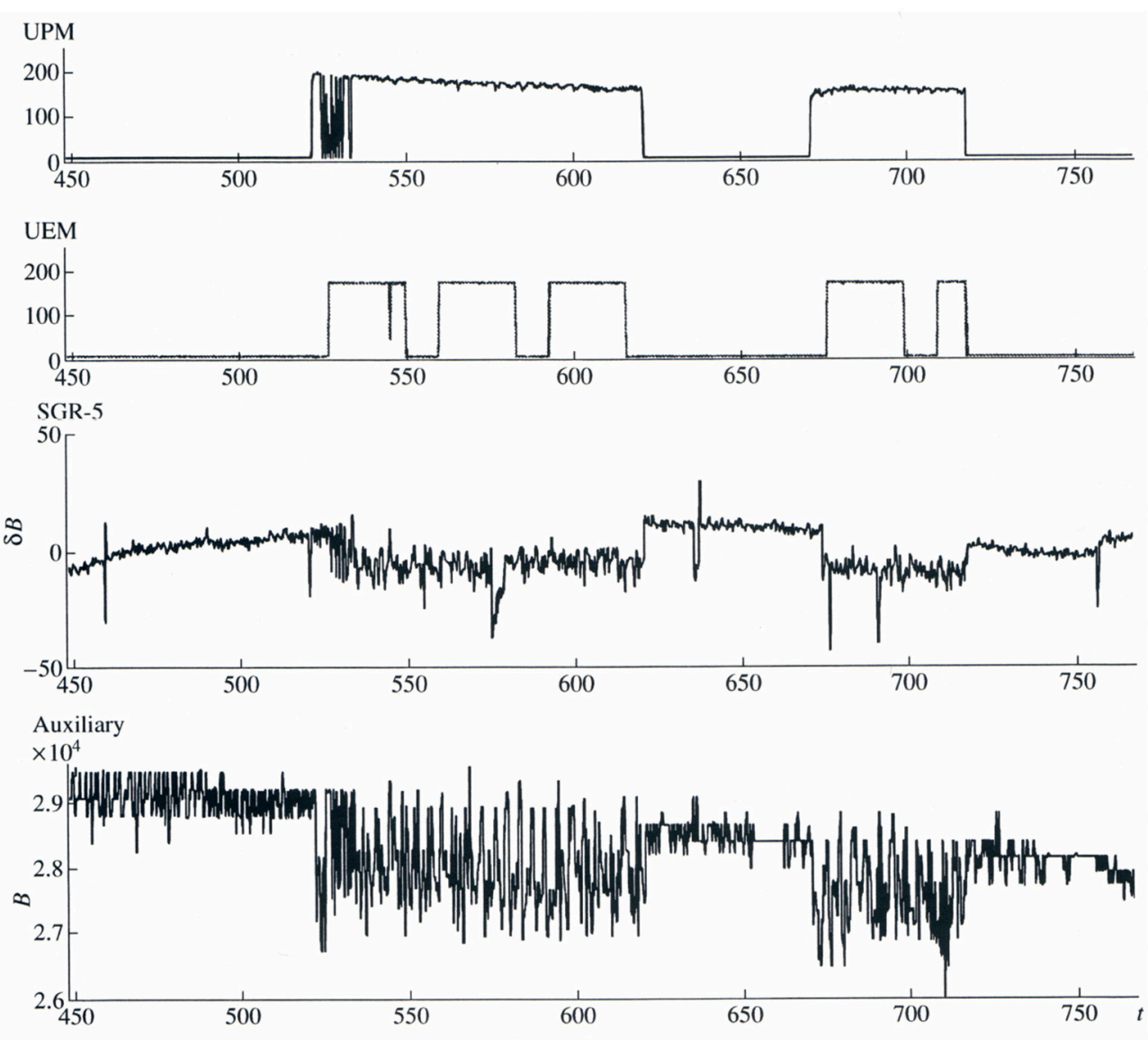

FIGURE 9 | Illustration of the diamagnetic cavity formation as seen in the time-dependent variations of the magnetic field. Horizontal axis - the time [s] lapsed from 04:37:02 UT, obtained during the xenon plasma injection on March 13, 1992 (orbit 1019). From top to bottom the UPM and UEM acceleration voltage [a.u.], deviation of the magnetic field strength [nT] from a mean level (SGR-5), and the service magnetometer total magnetic field [nT]. (Reprinted from Volokitin et al., 2000).

emission during the former interval and harmonics of this structure were also excited. During the latter injection the recorded spectra were more diverse, main emissions consisting of even harmonics of the cyclotron frequency $f_{c}$, dublets below $2 f_{c}$, or selectively excited emissions near $3 f_{c}$ and $4 f_{c}$. The authors interpreted the individual spectral peaks as emissions characteristic for synchrotron maser radiation created in a system of cold magnetoplasma and cold, weakly relativistic, very diluted electron beam.

Considering the electron beam under the F40K modulation as "strong," Kiraga (2003) brought a picture of HF emissions obtained during "weak" electron beam emissions in the S250 and S15.6 modulation modes. Evolution of spectra during the beam cycle, presence of modulation frequency harmonics and structures around the upper hybrid frequency are discussed as well as effects of antenna charging and electron current coupling between plasma and antenna. The electron beam injection as a tool for continuous monitoring of ambient plasma density by reception of emissions generated in ambient $\left(f_{p}, f_{u h}\right)$ frequency band is also discussed in the paper.

Budko et al. (2003) investigated HF waves excited by the pulsed electron injections and xenon plasma releases in the near zone. In lower altitude, subauroral dayside ionosphere they recorded emissions at the local plasma frequency $f_{p}, 2 f_{p}$, and $4 f_{c}$ during a weak electron injection alone. Later on during the simultaneous electron beam (S250) and xenon plasma (F0) injections, whistler mode wave excited at the electron beam modulation frequency $\sim 250 \mathrm{kHz}$ and resonances at the first three cyclotron frequency harmonics were present in the PRS3 frequency range (the local plasma frequency during the xenon plasma release was estimated by the authors to $\sim 16 \mathrm{MHz}$ which made detection of plasma resonance of an electron beam and background plasma impossible). During strong electron injection modulated by the $250 \mathrm{kHz}$ frequency, with only neutral xenon release, the dublet of local plasma and upper hybrid frequency peaks (reported by Kiraga et al., 1995 for $40 \mathrm{kHz}$ modulation) was clearly present together with whistler mode waves possibly up to the 6th to 8th harmonic of the electron beam modulation frequency. During nightside subauroral and middle latitude electron beam injection (F40K), resonant peaks near even electron cyclotron frequency harmonics $\left(2 f_{c}, 4 f_{c}, 6 f_{c}, 8 f_{c}\right)$ or combinations with the plasma frequency $\left(f_{p}, 2 f_{c}+f_{p}, 4 f_{c}+\right.$ $f_{p}, 6 f_{c}+f_{p}$ ) were recorded in the former case, plasma resonance harmonics only in the latter case. 
Recently, very detail analyses of the beam-plasma instability development, excitation of VLF/LF and HF waves, coupling of waves, particle heating and acceleration related to different APEX experiment configurations (injections through/opposite the xenon plasma cloud, quasi-perpendicular electron beam injections, injections of differently modulated electron beam - S250, F40K modes) have been performed in a series of papers by Baranets et al. $(2007,2012,2017)$ where the authors compared theoretically derived quantities with data obtained onboard IK-25 (VLF/HF emissions, electric and magnetic field fluctuations, plasma parameters) and remotely by MAGION3 (HF emissions, accelerated electron fluxes, thermal plasma parameters). Presented results reflect the development of a beam or beam-anisotropic instabilities in the ionospheric plasma, some of them being a consequence of more complex combination (nonlinear) mechanisms of interaction, developing during injection of a beam into a beam.

Baranets et al. (1999, 2000), and Oraevsky et al. (2001) analyzed waves generated during the DC and modulated electron beam injections in the near zone in a special dayside configuration when the electron beam was injected downward $\left(\alpha_{p e} \simeq 74-87^{\circ}\right)$ and, asynchronously to the UEM cycle (S250 modulation mode), the neutral xenon/xenon plasma (F0 mode) were released upward $\left(\alpha_{p i} \simeq 121-132^{\circ}\right)$, see also Figure 3. Beam-plasma discharge did not occur during this quasi-transverse injection, conditions for the beam-plasma instability development/suppression were investigated in these papers. Theoretical and numerical analysis of the observed data led the authors to the following conclusions:

- The beam plasma instability due to the electron beam injection $\left(\alpha_{p e} \simeq 74-87^{\circ}\right)$ excited ELF/VLF waves activity below the ion cyclotron frequency, growth of the magnetic component of VLF waves was observed. Increase of thermal spread of the beam electrons leads to the suppression (decay) of the excited VLF waves.

- Magnetic disturbances were recorded, caused by either the beam primary electrons or return currents associated with the beam-plasma instability.

- The electron beam injection was accompanied by strongly anisotropic plasma heating and modulation of the plasma (ion) flows in the vicinity of the satellite.

- For an unmodulated electron injection, the efficiency of plasma (beam) heating with respect to the transverse component decreases substantially when $\alpha_{p e}+\Delta \alpha^{\prime} / 2>90^{\circ}$ ( $\Delta \alpha^{\prime}$ is the electron beam effective pitch-angle width).

- The thermal flux of $\mathrm{O}^{+}$and $\mathrm{NO}^{+} / \mathrm{O}_{2}^{+}$ions decreased during the DC electron injections, probably as a consequence of the resonant coupling with the excited VLF waves.

- During the modulated beam-plasma interaction, the plasma resonance heating of $T_{e x}, T_{e y}$ components at the modulation frequencies corresponding to the $\mathrm{O}^{+}$and $\mathrm{NO}^{+} / \mathrm{O}_{2}^{+}$ion plasma frequencies were recorded.

Baranets et al. $(2007,2012,2014)$ studied modulated electron injections (S250 mode) performed in the same direction (upward, $\alpha_{p e}>90^{\circ}$ ) as the xenon plasma release. Experimental observations of the anomalous fluxes of fast charged particles, disturbances of quasi-steady and ELF/VLF magnetic field components, and thermal plasma ion fluxes have been considered using the IK-25 and MAGION-3 data obtained from two consecutive orbits with similar ionospheric plasma and injection parameters. Anomalously large disturbances $400-500 n T$ and fluxes of fast electrons were registered by MAGION-3 $(\sim 100 \mathrm{~km}$ from IK-25) during these injections. In their analysis the authors did not consider the xenon plasma cloud as a diamagnetic cavity in the nearest vicinity of the spacecraft, describing it as a hollow beam of about half a kilometer diameter. The electron beam current profile was considered as a hollow flow after several gyro-turns due to electrostatic repulsion, electron beam modulation was commented in the papers. In such experimental configuration (Figure 10A) the system of the electron beam nested in the xenon ion beam was axially asymmetric with respect to the magnetic field direction due to the small velocity of xenon ions $\left(v_{i z} / u \sim 3 \times 10^{4}\right)$, which was comparable to the velocity of the satellite $\left(v_{i z} / v_{s} \sim 1.5\right)$ moving at an angle to the magnetic field. The beam of heavy xenon ions injected at the pitch angles range up to $\Delta \alpha_{p i} \simeq 60^{\circ}$ with a maximum flux density within the angles $\Delta \alpha_{p i} \simeq 30^{\circ}$ would play the role of a damping layer for waves induced by the electron beam in the entire interaction region in the vicinity of the satellite. Main attention was paid to study the electromagnetic and longitudinal waves excitation in different frequency ranges and the energetic electron fluxes disturbed due to wave-particle interaction with whistler waves. The authors described dispersion relations for the whistler-mode wave excitations for the beam-driven electromagnetic instability and estimated its growth rate (in a weak beam approach).

Recent papers (Baranets et al., 2012, 2017), analysing the stationary electron beam injection (F40K modulation mode, $2 \mu$ s pulses) with simultaneous xenon neutral gas/plasma release (Figure 10B), reported a number of beam-plasma interaction effects both in the vicinity of the injecting satellite and in the far zone (subsatellite observations at distance about hundred kilometers, see also section 3.4.2). Based on thermal plasma ion fluxes, photometric, and wave data they found their correlation with beats of the density and velocity waves in the beam core in the high-frequency parallel and perpendicular wave fields. Theory and numeric estimations of correlation amplitudes are included. Onboard IK-25, sudden VLF amplification lasting 7$8 \mathrm{~s}$ was recorded. Analysis shows the burst was observed near the linear stability boundary for the slow beam mode excited due to a dissipation of beam kinetic energy. HF waves recorded by MAGION-3 were disturbed during the electron beam/xenon injections. The amplification of the HF wave amplitude in the range $\sim 0.6-1.1 \mathrm{MHz}\left(\omega_{c e}, \omega_{p e}, k_{0} u\right)$ and $2.0-2.3 \mathrm{MHz}(\sim$ $\left.2 k_{0} u\right)$ were correlated with the VLF burst at IK-25. Narrow bursts within the $2.20-2.3 \mathrm{MHz}$ range were modulated by the VLF-LF wave activity in accord with the xenon ion beam current $I_{b i}$. Stimulated soft electron fluxes also recorded by MAGION-3 were explained as disturbed due to the Cherenkov wave-particle interaction (acceleration/scattering) $-50-70 \mathrm{eV}$ electrons interacted with excited waves near $0.1 \mathrm{MHz}$, fluxes 
A

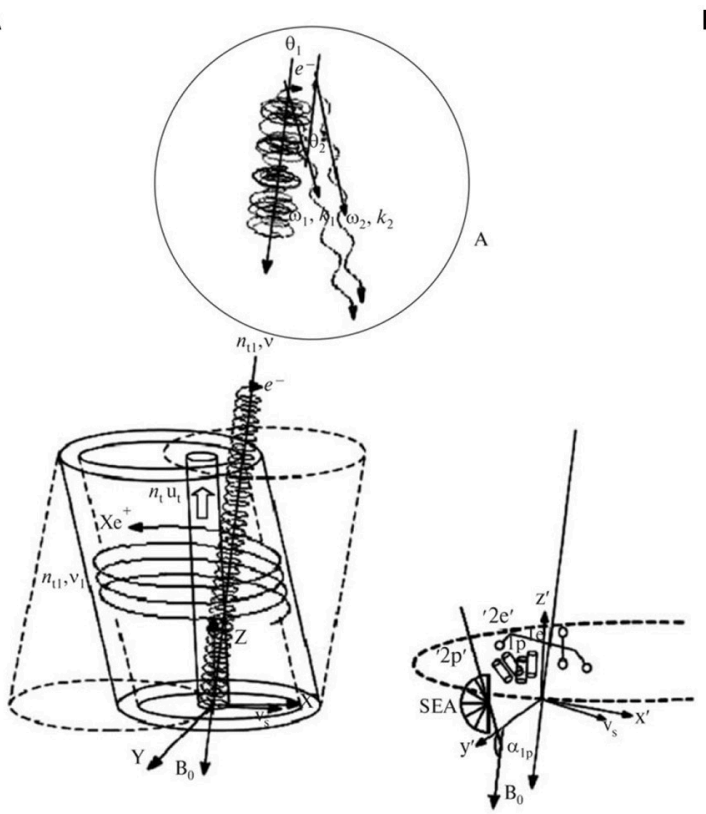

B

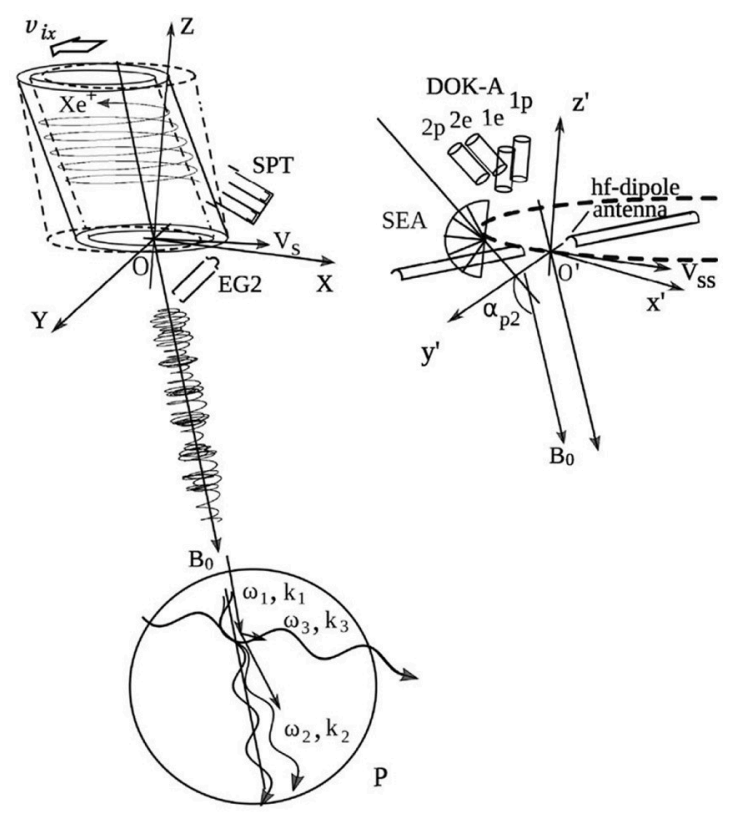

FIGURE 10 | (A) Electron beam injection ( $e^{-}$, modulation mode S250) directed through the hollow beam of xenon ions (Xe $\mathrm{e}^{+}$, no modulation F0) with the beams density and velocity $n_{b e}, v$, and $n_{b i}, v_{i}$, respectively. Release of the electron flow with the density and stream velocity $n_{e}, u_{e}$, for a compensation of the ion beam charge at the output of UPM is shown by the up arrow. Here, $B_{0}$ and $v_{S}$ are the directions of the quasi-steady magnetic field and the satellite velocity in the XYZ coordinate system, Z-axis points away from the Earth. Circle A-region of whistler-mode wave $(\omega, k)$ generation by the electron beam $e^{-}$at angle $\theta$ to the magnetic field. ("1e, 1," "2e, 2p") are two pairs of the DOK-A-S energetic particle sensors, SEA is the six-sector soft electron detector of the MPS/PPS spectrometer, $\alpha_{1 p}$ is the pitch-angle of the first sector registration in the x', y', z' MAGION-3 coordinate system. Injection configuration for orbits 201, 202. (Reprinted from Baranets et al., 2014). (B) Electron beam injection ( $e^{-}$, modulation mode F4OK) directed opposite to the hollow beam of xenon ions ( $\mathrm{Xe}^{+}$), disposition sketch of the electron gun (EG2) and stationary plasma truster (SPT) are shown in the IK-25 satellite coordinate system OXYZ. Sensors for the soft and fast charged particles belonging to the spectrometers MPS/PPS (SEA) and DOK-A-S ("1e, 1p," "2e, 2p"), respectively, and hf-dipole antenna (HF-receiver PRS-S) are shown in the subsatellite system Ox ${ }^{\prime} y^{\prime} z^{\prime}$, where $\alpha_{p 2}$ is a pitch-angle of soft electrons detected through the 2 nd sector of the SEA sensor aperture; $v_{i x}$ as well as $V_{S}$ and $V_{s S}$ are the inertia Xe-ion velocity shear and velocities of the satellite and subsatellite, respectively. Region $\mathrm{P}$ is a symbolic sketch of three-wave interaction. The distance OO' was about $\simeq 110 \mathrm{~km}$ for orbit 190. (Reprinted from Baranets et al., 2017, with permission from Elsevier).

with energies $>0.5 \mathrm{keV}$ could be stimulated by the beaminduced waves around $k_{0} u$ near the linear stability boundary for longitudinal waves.

\subsection{Distant Injection Effects 3.4.1. Energetic Particles}

Nemecek et al. (1996) and Prech et al. (2002) reported on observations of short intensive bursts of narrow-beam electrons onboard MAGION-3 during the electron injections from the main IK-25 satellite. The events were registered in the middle geomagnetic latitudes (INL $<55^{\circ}$ ) near local noon when the two satellites moved approximately along the same magnetic meridian at relative distances $64-550 \mathrm{~km}$. The localization of the burst observation to the altitudes from 800 to $1,500 \mathrm{~km}$ was probably a consequence of the local time favorable for the observation, the satellite orbit, and other parameters. The pitch angle distributions were narrow $\left(<10^{\circ}\right)$ and the electrons precipitated toward the atmosphere. The events lasted 4-10 $\mathrm{s}$ in the narrow MPS low-energy channel, but some events were doubled or tripled. The burst cross section was spatially limited to several tens of kilometers in the direction of the orbit and the electron energy extended to several hundred $k e V$, without measurable dispersion indicating proximity of the source. The events had different characteristics than electron microbursts already reported in the literature (for a short review see Prech et al., 2002). Examples of bursts registered by MAGION-3 are presented in Figure 11.

The main IK-25 satellite did not detect electron bursts of this kind as no other satellite orbiting in this region did but active electron injections from IK-25 were performed when the bursts were detected. The events of this kind were detected only during the UEM F40K mode operation and not during the S250 or other modulation mode injections. Neutral xenon or xenon plasma (UPM F0/F1000 modes) were simultaneously released so the quality of the satellite charge neutralization seems not to be important. Thirty four F40K-mode electron injections were scheduled on IK-25 during January and February 1992, but not all were $100 \%$ successful and with the MAGION-3 data coverage. The burst observation probability per session was about 0.25.

The energy-pitch angle properties of the bursts indicate according to Prech et al. (2002), that the bursts were created from low-temperature plasma or the beam emitted from the IK25 satellite by some acceleration process effective only in one direction with respect to the magnetic field (e.g., field-aligned 


\section{A MAGION-3 \& APEX 23-Jan-1992 (orbit 0431)}
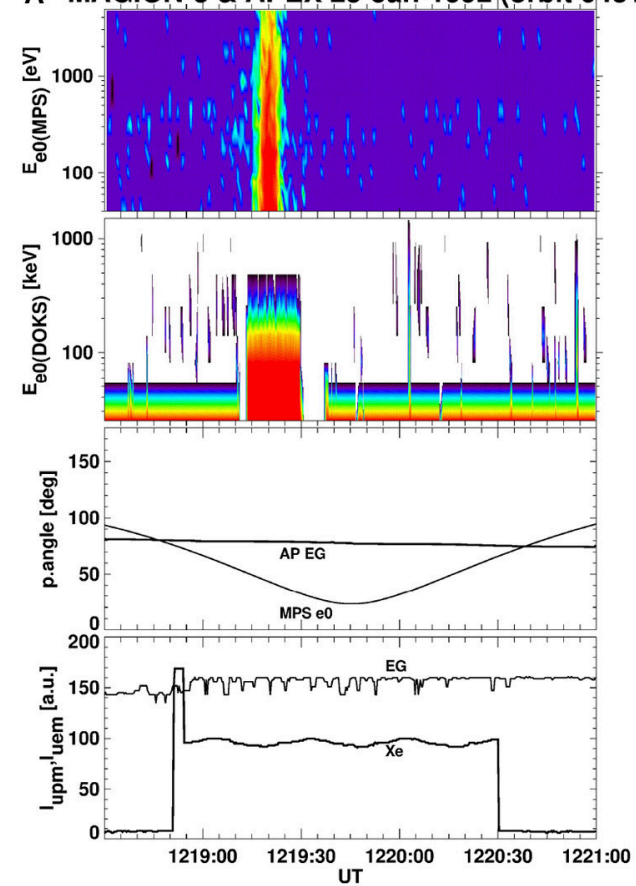

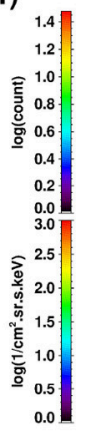

B

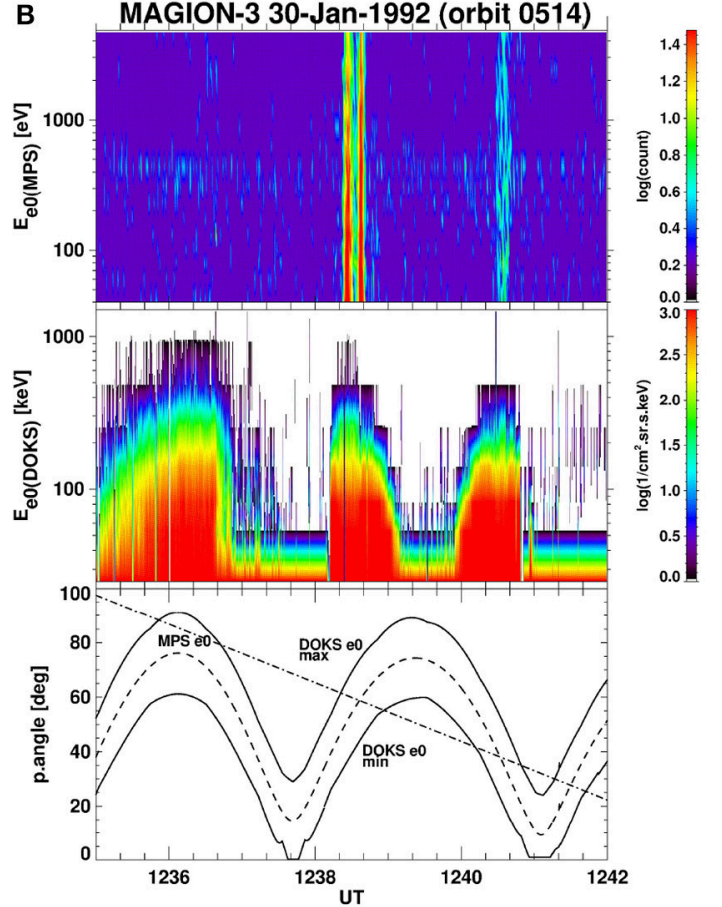

FIGURE 11 | Examples of single and multiple electron bursts registered onboard MAGION-3. (A) From top to bottom: MPS e0 channel dynamic spectrogram (to 5 keV, parallel to the satellite axis); DOK-S e0 channel spectrogram up to $1 \mathrm{MeV}$; IK-25 UEM electron beam pitch angle (AP EG) and the pitch angle of MPS e0 electrons; monitors of the IK-25 UEM and UPM injectors. (B) From top to bottom: MPS e0 and DOK-S e0 channel spectrograms; pitch angle of electrons registered by MPS eO (dashed line), the range of pitch angles covered by the DOK-S e0 channel (two thin min/max lines), and the approximate change of the electron burst pitch angle with time (dash-dotted line). (Originally published in Prech et al., 2002, distributed under the Creative Commons Attribution 3.0 License).

potential drop), the spread of the pitch angles being caused by the original thermal velocity distribution. The authors were not in favor of the other possible source-ring current electrons released by some mechanism induced by particles emitted from IK-25. Questions if the beam-plasma interaction is able to accelerate the electrons to the observed energies, how the observed electrons moved across the magnetic field lines, and why the electron bursts were observed only in part of the active experiments are further discussed in the paper.

Baranets et al. $(2012,2014)$ reported on similar kind of fast electron fluxes (MAGION-3/DOK-S). The bursts had highly fluctuating wide (scattered) pitch-angle distribution, the electrons were simultaneously detected by both the DOK$S$ electron channels. The bursts were observed during the UEM S250 electron injection mode different from the previous paragraph case. According to the authors electromagnetic waves were excited via the electron-cyclotron resonance with the beam and subsequently scattered in a wave-particle interaction (Cherenkov resonance) giving rise to the energetic electrons distantly observed by MAGION-3.

\subsubsection{HF Waves}

Rothkaehl et al. (1995) reported simultaneous observations of RF emissions on the mother IK-25 satellite and the MAGION3 subsatellite during a downward UEM modulated electron beam injection (F40K mode, $2 \mu$ s pulses, only neutral xenon released by UPM during this case). The spacecraft separation was about $200 \mathrm{~km}$. Strong electrostatic emissions at the upperhybrid plasma frequency and its harmonics at the main spacecraft and spikes in the HF frequency range on MAGION-3 (see Figure 12) were simultaneously detected. The first emission peak was at frequency $2.15 \mathrm{MHz}$ and three harmonics were also recorded. The authors concluded the electromagnetic emission of the oscillating current created by the injected electron beam was observed, the base frequency attributed to the electron beam current properties and the half-width of the emission line $\sim$ $50 \mathrm{kHz}$ referred to the electron gun pulse repetition frequency.

\subsubsection{Ground VLF Transmitter Spectral Broadening}

Spectral broadening of signals from ground-based VLF transmitters $(16.5,12.6,11.333 \mathrm{kHz}$ carriers) was observed onboard MAGION-3 during the modulated electron beam injection from the main IK-25 satellite (Oraevsky et al., 1994). The broadening of order of 300-500 Hz was apparently correlated with the 2-s cycle of the UEM gun pulses modulated in the range $30.5-31,250 \mathrm{~Hz}$ (2 $\mu$ s length) while no effects were observed for modulation frequencies $62.5,125$, and $250 \mathrm{kHz}$ (Figure 13). The observations were made in the middle latitude ionosphere at altitudes $1,175-1,580 \mathrm{~km}$, the distance between the two satellites was about $250 \mathrm{~km}$. Scattering of whistler-mode waves into quasi-electrostatic waves by periodic small-scale plasma inhomogeneities or ELF plasma turbulence created by 
A

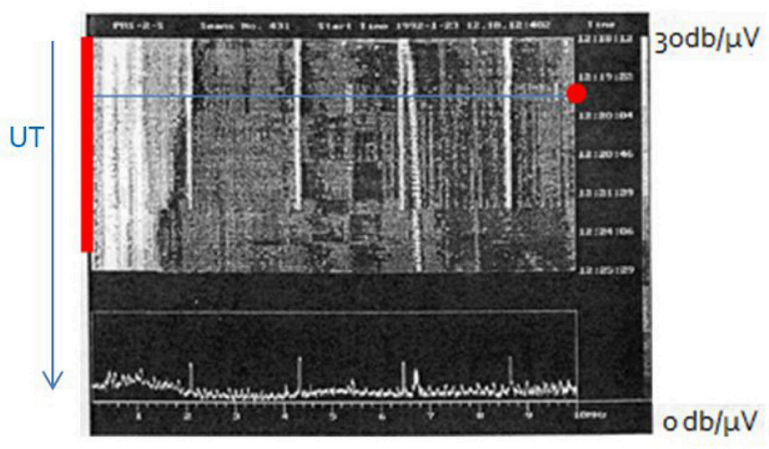

B

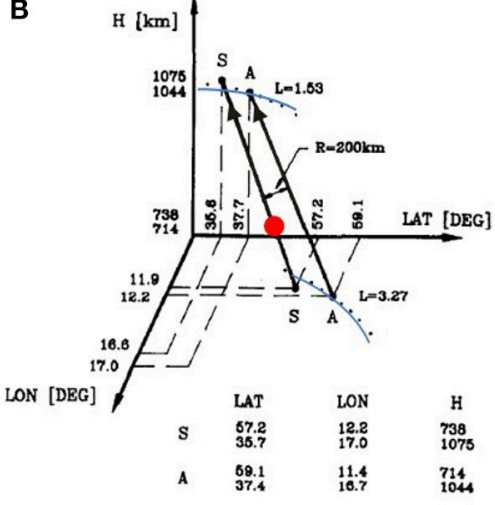

FIGURE 12 | HF waves registered onboard MAGION-3 durin the electron beam emission from IK-25. (A) Shows a sequence of radio spectra (PRS-2-S, vertical axis for time) along the orbit 0431. Emission intensity from black $0 \mathrm{~dB} / \mu \mathrm{V}$ to white $30 \mathrm{~dB} / \mu \mathrm{V}$ with nine equidistant levels. During the IK-25 electron beam injection (marked with the red bar) spiky emissions at $\sim 2.1 \mathrm{MHz}$ and harmonics were registered. Bottom plot represents a single spectrum (12:19:37 UT, blue line cut). (B) Displays the relative position of the $\mathrm{IK}-25$ satellite (A) and MAGION-3 (S) in the geographic coordinates. Their relative distance during injection was about $200 \mathrm{~km}$. The dotted lines present the geomagnetic field lines. The injection started at 12:18 UT (altitude 714 km) and finished at 12:24:30 UT (alt. 1,044 km). The red dots (both panels) mark the electron burst reported by Prech et al. (2002) in MAGION-3 data. (Reprinted from Rothkaehl et al., 1995, with permission from Elsevier).

the pulse-modulated electron beam were discussed as possible causes of the observed phenomenon. The authors compared the observation with previous rocket and space shuttle experiments and discussed both local (near the injection source) and remote (e.g., ionizing effect of the electron beam at the ionospheric E-region heights) mechanisms of plasma irregularities creation that had been suggested in literature.

\section{5. lonospheric Heating Experiments}

Magnetosynchronous active experiments belong to a different group of active experiments in space plasma. One of possible approaches to investigate evolution of physical phenomena along a geomagnetic flux tube after a disturbance injection could be to use a special satellite orbit which runs in parallel with the geomagnetic field line for a sufficient time. These orbits are called magnetosynchronous and a concept of such active experiment in frame of the APEX programme was introduced by Ruzhin and Vaskov (1992). For the APEX orbit with inclination $\sim 80^{\circ}$ the magnetic meridian fell in the orbital plane about twice per day. The experiment was realized using the Dushanbe ionospheric heater for several IK-25 flyover orbits both near perigee and apogee.

Oraevsky et al. (1998b) reported on observation of the plasma barrier transparency effect (passing of a shortwave signal below the $\mathrm{f}_{0} \mathrm{~F} 2$ frequency across the $\mathrm{F} 2$ layer, in the particular case for the heater frequency $5.98 \mathrm{MHz}$ the barrier thickness was estimated $\sim 100 \mathrm{~km}$ ). The ballistic transport mechanism was suggested by the authors to explain detection of increased noise at frequencies around $6 \mathrm{MHz}$ from PRS-3 data while the IK25 satellite was magnetically connected to the heated part of the ionosphere. Oraevsky et al. (1998a) reported on similar results for the APEX and CORONAS satellites and the SURA ionospheric heating facility, more recently the experiment was repeated with the DEMETER satellite and the SURA heater (Zhang et al., 2016).
Variations of electron temperature and density near IK-25 apogee observed when magnetically connected to an ionospheric spot heated by the Dushanbe facility were reported and analyzed by Oraevsky et al. (1998b). Propagation of disturbances from locally artificially heated ionosphere into magnetosphere was modeled e.g., by Ruzhin and Vaskov (1992) and recently by Borisov et al. (2015).

\section{COMPARISON WITH OTHER EXPERIMENTS AND GENERAL DISCUSSION}

The analysis of the KM-10 floating probe potential and PEAS charge particle spectra has shown small charging and good neutralization during electron beam emissions from the IK-25 satellite. A larger spacecraft surface and methods of electron beam charge compensation made a difference comparing the electron beam emissions in a similar range of altitudes from the G-60-S sounding rocket (Managadze et al., 1988) or even at lower altitudes (SCEX-3, Mullen et al., 1991) where high body charging was observed.

Measurements of angular-energetic spectra of charged particles were rare prior to the APEX project. Previous sounding rockets and spacecraft active experiments included simple electrostatic analyzers that did not allow detail studies of pitchangle distributions of charged particles in the vicinity of the beam emitting body. The space shuttle Spacelab-1/SEPAC and TSS-1/SETS active experiments (e.g., Burch, 1986; Watermann et al., 1988; Oberhardt et al., 1993) provided electron angular distributions with high time resolution but related pitch-angle distributions were not discussed. Moreover, configurations of these experiments were different from APEX. Most of the shuttle surface was covered by non-conducting ceramic tiles. The electron spectrometers were mounted on a platform inside 


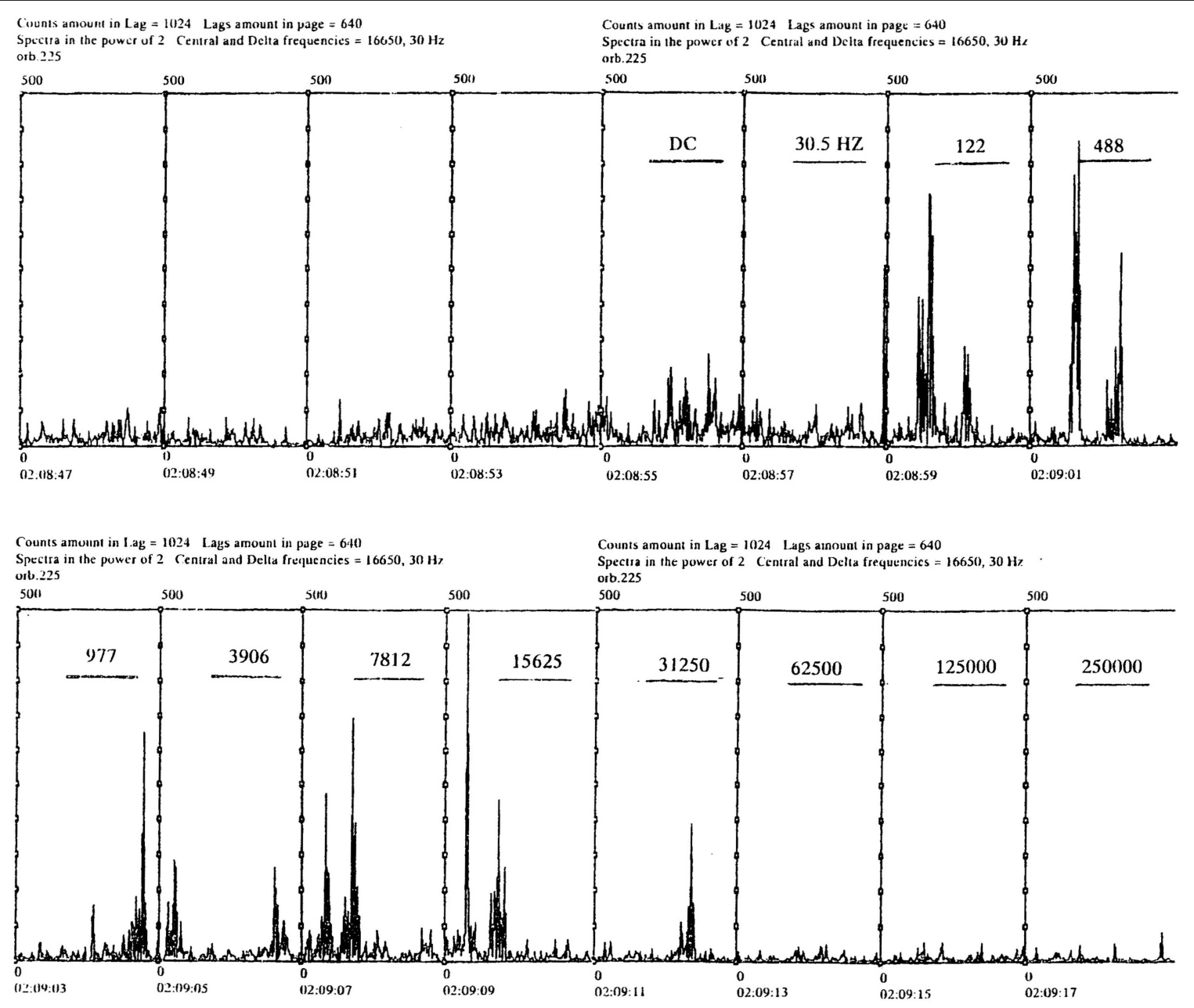

FIGURE 13 | Wave intensity registered by MAGION-3 at the frequency $16,650 \mathrm{~Hz}$, i.e., $150 \mathrm{~Hz}$ away from the VLF transmitter carrier. The electron beam pulses are shown as horizontal bars with the modulation frequency indicated. (Reprinted from Oraevsky et al., 1994, with permission from Elsevier).

the shuttle payload bay where at least half of space was shielded. The angular distribution of the return current electrons during electron beam emissions did not show flux decrease in the shielded sectors indicating the origin of electrons near the platform. Their angular distribution depended more on the sector view angles against the platform normal and the direction toward the electron gun. For pitch-angles close to zero the electron energy was limited to about primary beam energy while for pitch-angles reaching $\sim 80^{\circ}$ intense electron flux was observed up to the spectrometer upper range $(\approx 2.5$ times the beam energy). In the APEX experiments, return electrons with energies of the primary beam and above were not observed in the IK-25 vicinity.

We consider important our observations of the short highenergy electron bursts in the remote zone by MAGION-3 during the electron injections. To our knowledge these observations have not been confirmed by other experiments yet, actually because no other experiment with two-point observations similar to APEX has been performed up today. Comprehensive wave and particle measurements were realized during the electron beam emissions from the STS-3 and Spacelab-2 space shuttle platforms (e.g., Gurnett et al., 1986; Banks et al., 1987; Bush et al., 1987) using the independent Plasma Diagnostic Package (PDP; mounted on a manipulator arm or free-flying, resp.) but they were made in the near zone at distance $\sim 10^{1}-10^{2} \mathrm{~m}$. The MAGION-3 HF wave measurements also remain unconfirmed for the same reasons.

Kawashima and Akai (1986) reported on waves registered onboard the satellite JIKIKEN (EXOS-B) on an elongated orbit with apogee $\sim 25,000 \mathrm{~km}$ that were excited during electron beam injections (much weaker than later used in the space shuttle or APEX experiments). They also observed waves near upperhybrid or plasma frequencies, electron-cyclotron frequency, and LF frequencies related to the electron beam instrumental modulation. Accompanying harmonics were supposed to be generated instrumentally due to the saturation of the signal level. Harmonics of the plasma frequency in the APEX data might have the same instrumental origin which was not discussed by Budko 
et al. (2003), or they could truly exist due to the beam bunching as noted by Kiraga et al. (1998).

From their analysis of the return electron spectra, Nemecek et al. (1997) and Budko et al. (2003) deduced a presence of electric fields of a strength up to $100 \mathrm{~V} / \mathrm{m}$ in the vicinity of the spacecraft that was sometimes questioned by a part of the scientific community and would require further study. Anyway, according to the 3D computer simulation by Pritchett (1991) electric fields of the order of tens of volts per meter can be expected during an electron beam injection in similar conditions.

Complexity of the IK-25 design (structure, surfaces, etc.) and a lack of related engineering data have not allowed to synthesize a more realistic model of the electron beam and xenon plasma injections and return currents in the near zone including the satellite influence that would help to interpret the observational data. Earlier papers related to the APEX wave observations (Kiraga et al., 1998; Budko et al., 2003; Kiraga, 2003) did not aim to establish a comprehensive theory of wave generation by the APEX electron beam and xenon plasma injections but in their analysis of observed data they rather referred to previous theoretical treatments of beam-plasma interaction which is non-linear in the APEX case. Full understanding of the coexistence and competition between various instabilities requires dedicated simulations which would take into account many unique characteristics of the APEX experimental setup, but such APEX-related simulation models have not been developed. Baranets et al. $(2007,2012,2017)$ presented non-linear theoretical analysis of beam-plasma interaction for systems of electron beam nested in or opposite to the ion beam that were related to the APEX injection configurations. Fast electrons from the MAGION-3 observations were explained as a result waveparticle interaction: whistler waves excitation by the electroncyclotron resonance and subsequent scattering. To verify their results, a detail knowledge on wave propagation ( $k$ vector) would be useful, but such data were not gathered in the APEX project.

The APEX project suffered from a relatively short life-time of active devices UEM-2 and UPM. The active experiments with charge particle beam injections were performed only during the first six months after the launch, recurrent technical issues precluded to obtain complementary data during the second half year or to repeat unsuccessful active experiment configurations. Above that, some operational and science information was not available (fast beam current monitoring, optical diagnostics, cold plasma spectra), the field and wave instruments did not provide complete information. Technical difficulties connected with the precise control of the MAGION-3 orbit did not allow intended near- and mid-zone observations during the active experiments while the hundred-kilometers satellite distances were preferred for two-point passive measurements in the auroral region. Last but not least, the available computer network communication means limited daily supervision by scientists, quick preliminary data analysis, and feedback toward the main satellite operation control-reasonable demands of a contemporary active experiment.

Many questions connected to the APEX active injections have not been answered, e.g., what was the size and structure of the electron acceleration region or what conditions allowed the observed acceleration up to relativistic energies. A new mission could provide multi-point measurements in medium and distant zones of injection $\left(1-10^{3} \mathrm{~km}\right)$ using a constellation of nanosatellite probes that would allow the study of waves growth and propagation and electron acceleration simultaneously at different distances along and perpendicular to the beam. If feasible, the orbit (active experiment parts) should be chosen so as the emission pitch-angle, altitude, latitude, L shell, and local time are not closely bounded, with sufficient coverage of parameter space and repeatability of emission parameter subsets. Wave-particle correlators and ion analyzer with mass selection would be a useful extension of the APEX scientific payload. A new experiment should be also equipped with a 3D measurement of LF and HF electric and magnetic fields. Such data, when processed using the latest analysis methods to obtain wave propagation direction (e.g., Santolik et al., 2003), polarization (Santolik et al., 2016; Taubenschuss and Santolik, 2018), and HF mode identification (Santolik and Parrot, 2006), can give a new insight into the beam-plasma interaction processes. Last but not least, Kiraga (2003) discussed also usage of the APEX type electron injection and HF waves analysis for monitoring density of cold plasma.

\section{CONCLUSIONS}

The broadly focused APEX active experiments have an important place in the long line of active space experiments. They brought new results or they complemented results of previous projects made using sounding rockets, space shuttles, or scientific/technological satellites in ionosphere and magnetosphere. The main achievements of the active experiments within the APEX project could be listed as

- successful neutralization of the spacecraft charge during electron beam emissions by xenon/xenon plasma releases at altitudes $500-1,000 \mathrm{~km}$,

- analysis of ELF/VLF and HF wave activity near the electronbeam emitting satellite,

- first in-situ measurements in the distant zone of injection (MAGION-3 subsatellite),

- MAGION-3 observation of electron bursts accelerated to energies of several hundred $k e V$ during the electron beam injections from the main satellite.

The mother-daughter (multi-point) satellite projects were quite rare till the end of the twentieth century and we can treat the APEX project also as the pathfinder in this methodology and the MAGION series satellites as predecessors of contemporary micro, nano, and cube satellites. Repeating the APEX project with state-of-the-art scientific 
equipment and a fleet of small satellite probes around would certainly bring new scientific achievements and help to answer some still open questions in the field of space plasma.

\section{AUTHOR CONTRIBUTIONS}

YR, VD, ZN, and JS contributed to the conception and design of the APEX project. LP wrote the first draft of the manuscript. YR wrote substantial parts of several sections of the manuscript. LP, JS and ZN selected and prepared figures to be reprinted in this review. All authors contributed to

\section{REFERENCES}

Afonin, V. V., Grechnev, K. V., Ershova, V. A., Roste, O. Z., Smirnova, N. F., Smilauer, J., et al. (1994). Ion composition and temperature of ionosphere at maximum of the 22nd solar activity cycle from satellite Interkosmos-24 (ACTIVE project). Kosm. Issled. 32, 82-94. in Russian.

Artsimovich, L. A., Andronov, I. M., Yesipchuk, Y. V., and Barsukov, I. A. (1974). Development of a steady plasma engine (SPE) and its testing onboard the "Meteor" artificial satellite. Kosm. Issled. 12, 451-468. in Russian.

Banks, P. M., Raitt, W. J., White, A. B., Bush, R. I., and Williamson, P. R. (1987). Results from the vehical charging and potential experiment on STS-3. J. Spacecr. Rockets 24, 138-149. doi: 10.2514/3.25887

Baranets, N., Ruzhin, Y., Dokukin, V., Ciobanu, M., Rothkaehl, H., Kiraga, A., et al. (2017). Injection of $40 \mathrm{kHz}$-modulated electron beam from the satellite: I. beam-plasma interaction near the linear stability boundary. Adv. Space Res. 59, 2951-2968. doi: 10.1016/j.asr.2017.03.030

Baranets, N., Ruzhin, Y., Erokhin, N., Afonin, V., Vojta, J., Smilauer, J., et al. (2012). Acceleration of energetic particles by whisler waves in active space experiment with charged particle beams injection. Adv. Space Res. 49, 859-871. doi: 10.1016/j.asr.2011.12.001

Baranets, N., Ruzhin, Y., Erokhin, N., Afonin, V., Vojta, J., Smilauer, J., et al. (2014). Resonance effects of wave-particle interactions during artificial charged particle beam injections in an ionospheric plasma. Kosm. Nauka Technol. 20, 3-26. in Russian. doi: 10.15407/knit2014.05.003

Baranets, N. V., Ruzhin, Y. Y., Afonin, V. V., Oraevsky, V. N., Pulinets, S. A., Dokukin, V. S., et al. (1999). Active experiment in space for investigation of beam plasma interactions. Results of the APEX project. Adv. Space Res. 24, 981-984. doi: 10.1016/S0273-1177(99)00561-X

Baranets, N. V., Ruzhin, Y. Y., Afonin, V. V., Oraevsky, V. N., Pulinets, S. A., Dokukin, V. S., et al. (2000). Electron beam injection quasilateral to the geomagnetic field from the data of Intercosmos-25 satellite: APEX project. Kosm. Nauka Technol. 6, 49-62. in Russian. doi: 10.15407/knit2000.05.049

Baranets, N. V., Sobolev, Y. P., Ciobanu, M., Vojta, J., Smilauer, J., Klos, Z., et al. (2007). Development of beam-plasma instability during the injection a lowenergy electron beam into ionospheric plasma. Plasma Phys. Rep. 33, 995-1013. translated from Russian. doi: 10.1134/S1063780X07120057

Borisov, N., Ryabova, N., and Ruzhin, Y. (2015). Dynamics of plasma density perturbations in the upper ionosphere and the magnetosphere under the action of powerfull HF radio waves. J. Atmos. Solar Terr. Phys. 134, 102-108. doi: 10.1016/j.jastp.2015.09.018

Budko, N. I., Prutenskii, I. S., Pulinets, S. A., Dokukin, V. S., Shutte, N. M., Plokhova, O. A., et al. (2003). Some features of the plasma turbulence region in the active experiments onboard the APEX satellite. Geomagnet. Aeron. 43, 335-344. translated from Russian.

Burch, J. L. (1986). Space plasma physics results from Spacelab-1. J. Spacecr. Rockets 23, 351-335. doi: 10.2514/3.25115

Bush, R. I., Reeves, G. D., Banks, P. M., Neubert, T., Williamson, P. R., Raitt, W. J., et al. (1987). Electromagnetic-fields from pulsed electron-beam experiments in space - Spacelab-2 results. Geophys. Res. Lett. 14, 1015-1018. doi: $10.1029 /$ GL014i010p01015 the manuscript revision, read, and approved the submitted version.

\section{FUNDING}

The work of LP, ZN, and JS was supported by the Czech Science Foundation contract 17-06065S.

\section{ACKNOWLEDGMENTS}

We thank to Jiri Simunek for providing the MAGION-3 to IK-25 orbital delay data.

Cambou, F., Dokukin, V. S., Ivchenko, V. N., Managadze, G. G., Migulin, V. V., Nazarenko, O. K., et al. (1975). The Zarnitsa Rocket Experiment on Electron Injection. Space Research XV. Berlin: Akademie-Verlag.

Cambou, F., Dokukin, V. S., Lavergnat, J., Pellat, R., Reme, H., Saintmarc, A., et al. (1980). General description of the ARAKS experiments. Ann. Geophys. 36, 271-283.

Choueiri, E. Y., Oraevsky, V. N., Dokukin, V. S., Volokitin, A. S., Pulinets, S. A., Ruzhin, Y. Y., et al. (2001). Observations and modeling of neutral gas releases from the APEX satellite. J. Geophys. Res. 106, 25673-25681. doi: 10.1029/2001JA000040

Dokukin, V. S. (1992). "The APEX project scientific facility orbital complex," in APEX Project. Scientific Purposes, Simulation, Technique and Equipment of Experiment, eds V. N. Oraevsky and Y. Y. Ruzhin (Moscow: Nauka), 16-29. in Russian.

Dokukin, V. S., Mishin, E. V., Markeev, A. K., Milinevsky, G. P., Mishin, E. V., Moisya, R. I., et al. (1981). Results of Zarnitsa-2, a rocket experiment on artificial electron beam injection in ionosphere. Adv. Space Res. 1, 5-15. doi: 10.1016/0273-1177(81)90268-4

Grandal, B. (ed.). (1982). Artificial Particle Beams in Space Plasma Studies. NATO Adv. Study Inst. Ser. B: Physics. New York, NY: Plenum Press.

Gurnett, D. A., Kurth, W. S., Steinberg, J. T., Banks, P. M., Bush, R. I., and Raitt, W. J. (1986). Whistler-mode radiation from the Spacelab-2 electron beam. Geophys. Res. Let. 13, 225-228. doi: 10.1029/GL013i003p00225

Haerendel, G., and Sagdeev, R. Z. (1981). Artificial plasma jet in ionosphere. $A d v$. Space Res. 1, 29-46. doi: 10.1016/0273-1177(81)90270-2

Hausler, B., Treumann, R. A., Bauer, O. H., Haerendel, G., Bush, R., Carlson, C. W., et al. (1986). Observations of the artifically injected porcupine xenon ion beam in the ionosphere. J. Geophys. Res. 91, 287-303. doi: 10.1029/JA091iA01p00287

Hess, S., Inguimbert, V., Siguier, J., Payan, D., Gerhard, A., DAccolti, G., et al. (2016). "Plasma current collection of high voltage solar array: numerical investigation," in 14th Spacecraft Charging Technology Conference SCTC 2016, ESA/ESTEC (Noordwijk). Available online at: https://hal.archives-ouvertes.fr/ hal-01371932/document

Izhovkina, N. I., Prutensky, I. S., Pulinets, S. A., Erokhin, N. S., Mikhailovskaya, L. A., Klos, Z., et al. (2009). Plasma inhomogeneities in the topside ionosphere in the region of the geomagnetic equator and wave radiation according to the APEX satellite data. Geomagn. Aeron. 49, 210-218. translated from Russian. doi: 10.1134/S0016793209020108

Kawashima, N., and Akai, K. (1986). JIKIKEN electron beam measurements of the plasmapause. Adv. Space Res. 6, 341-350. doi: 10.1016/0273-1177(86)90315-7

Kiraga, A. (2003). On the performance of a cylindrical dipole antenna in diagnostics of wave phenomena in space plasma. Adv. Space Res. 32, 429-440. doi: 10.1016/S0273-1177(03)90284-5

Kiraga, A., Klos, Z., Oraevsky, V. N., Dokukin, V. S., and Pulinets, S. A. (1995). Observation of fundamental magnetoplasma emissions excited in magnetosphere by modulated electron beam. Adv. Space Res. 15, 21-24. doi: 10.1016/0273-1177(95)00004-X

Kiraga, A., Klos, Z., Oraevsky, V. N., Dokukin, V. S., and Pulinets, S. A. (1998). "Electromagnetic emissions in the ionosphere - pulsed electron beam system," in Measurement Techniques in Space Plasmas: Fields, Vol. 103 of Geophysical 
Monograph, eds R. F. Pfaff, J. E. Borovsky, and D. T. Young, (Washington, DC: AGU), 185-191.

Klos, Z., Gdalevich, G. I., Mikhailov, J., Gousheva, M., and Bankov, L. (1998). VLF plasma waves observed during the xenon gas releasees on the ACTIVE satellite. Adv. Space Res. 21, 717-722. doi: 10.1016/S0273-1177(97)01 011-9

Lizunov, G., Volokitin, A., and Blazhko, I. (2002). Dynamics and relaxation of an artificial electron beam. Adv. Space Res. 29, 1391-1396. doi: 10.1016/S0273-1177(02)00192-8

MacDonald, E. A., Borovsky, J. E., Larsen, B., and Dors, E. (2012). A Science Mission Concept to Actively Probe Magnetosphere-Ionosphere Coupling. Decadal Survey in Solar and Space Physics papers. (Washington, DC: The National Academies Press).

Managadze, G., Balebanov, V. M., Burchudladze, A. A., Gagua, T. I., Leonov, N. A., Lyakhov, S. B., et al. (1988). Potential observations of an electron-emitting rocket payload and other related plasma measurements. Planet. Space Sci. 36, 399-410. doi: 10.1016/0032-0633(88)90128-6

Mikhailov, Y. M., Oraevsky, V. N., Sobolev, Y. P., Dokukin, V. S., Kapustina, O. V., and Afonin, V. V. (1998). Waves generated in the vicinity of xenon plasma gun in the APEX experiment. Adv. Space Res. 21, 713-716. doi: 10.1016/S0273-1177(97)01010-7

Mikhailov, Y. M., Oraevsky, V. N., Sobolev, Y. P., Dokukin, V. S., Kapustina, O. V., Shibaev, I. G., et al. (2000). Waves generated in the vicinity of xenon plasma gun in the APEX experiment. Phys. Chem. Earth (C) 25, 67-70. doi: 10.1016/S1464-1917(99)00039-2

Mishin, E. V., Rozhanskii, V. A., and Ruzhin, Y. Y. (1988). Influence of "short-circuiting" effect on the dynamics of an artificial comet (the AMPTE experiment). JETP Lett. 47, 31-34. translated from Russian. translated from Russian

Mullen, E. G., Gussenhoven, M. S., Hardy, D. A., Murphy, G. P., Lloyd, J. W. F., Slutter, W., et al. (1991). A spacecraft charging study on the scex 3 rocket. IEEE Trans. Nuclear Sci. 38, 1622-1628. doi: 10.1109/23.124154

Nemecek, Z., Safrankova, J., and Prech, L. (1993). A behavior of electron and ion energy and angular distribution during the active APEX experiment. Adv. Space Res., 13, 113-116. doi: 10.1016/0273-1177(93)90058-J

Nemecek, Z., Safrankova, J., and Prech, L. (1996). Charged particle behaviour during the active phase of the APEX experiment. J. Geomagn. Geoelectr. 48, 157-164. doi: 10.5636/jgg.48.57

Nemecek, Z., Safrankova, J., Prech, L., Simunek, J., Smilauer, J., Gringauz, K., et al. (1997). Artificial electron and ion beam effects: active plasma experiment. J. Geophys. Res. 102, 2201-2211. doi: 10.1029/95JA03571

Nemecek, Z., Safrankova, J., Santolik, O., Simunek, J., Smilauer, J., Fischer, S., et al. (1994). "Two point measurement of energy distribution relaxation: APEX project," in Solar-Terrestrial Energy Program: Initial Results From Step Facilities And Theory Campaigns, Vol. 5 of COSPAR Colloquia Series, eds D. N. Baker, V. O. Papitashvili, and M. J. Teague (Oxford: Pergamon Press Ltd.), $159-162$.

Neubert, T., and Banks, P. M. (1992). Recent results from studies of electron beam phenomena in space plasmas. Planet. Space Sci. 40, 153-183. doi: 10.1016/0032-0633(92)90055-S

Oberhardt, M. R., Hardy, D. A., Thompson, D. C., Raitt, W. J., Melchioni, E., Bonifazi, C., et al. (1993). Positive spacecraft charging as measured by the shuttle potential and return electron experiment. IEEE Trans. Nuclear Sci. 40, 1532-1541.

Oraevskii, V. N., Choueiri, E. Y., Dokukin, V. S., Volokitin, A. S., Pulinets, S. A., Ruzhin, Y. Y., et al. (1999). APEX: Neutral xenon release experiments. Geomagn. Aeron. 39, 582-591. translated from Russian.

Oraevsky, V. N., Chmyrev, V., Shibaev, I., Dokukin, V., Sobolev, Y., Shklyar, D., et al. (1994). Effects of artificially injected electron beams on the characteristics of ground VLF transmitter signals in the ionosphere. J. Atmos. Terr. Phys. 56, 423-431. doi: 10.1016/0021-9169(94)90223-2

Oraevsky, V. N., Pulinets, S. A., Bud'ko, N. I., Prutensky, I. S., Vaskov, V. V., Klos, Z., et al. (1998a). Emissions stimulated in the upper ionosphere by the SURA heating facility. Adv. Space Res. 21, 671-675. doi: 10.1016/S0273-1177(97)01002-8

Oraevsky, V. N., Ruzhin, Y. Y., Dokukin, V. S., Kanonidy, K. D., Singh, B. P., and Lakhina, G. S. (1998b). "Modification natural and manmade EM environment due to ionospherical plasma barrier transparency for groundbased transmiter emission," in Magnetospheric Research With Advanced Techniques, Vol. 9 of COSPAR Colloquia Series, eds R. L. Xu and A. T. Y. Lui (Oxford: Pergamon), 91-95.

Oraevsky, V. N., Ruzhin, Y. Y., Dokukin, V. S., and Morozov, A. I. (2003). Dynamics of quasineutral plasma beams and the structure of the beam-induced disturbances in ionospheric plasma. Plasma Phys. Rep. 29, 267-275. translated from Russian. doi: 10.1134/1.1561121

Oraevsky, V. N., Ruzhin, Y. Y., Dokukin, V. S., and Volokitin, A. S. (1992). "APEX project," in APEX Project. Scientific Purposes, Simulation, Technique and Equipment of Experiment, eds V. N. Oraevsky and Y. Y. Ruzhin (Moscow: Nauka), 6-16. in Russian.

Oraevsky, V. N., Sobolev, Y. P., Zhuzgov, L. N., Afonin, V. V., and Baranets, N. V. (2001). Magnetic field excitation during electron beam injection from the Intercosmos-25 satellite (APEX). Plasma Phys. Rep. 27, 322-329. translated from Russian. doi: 10.1134/1.1364551

Oraevsky, V. N., and Triska, P. (1993). Active plasma experiment - project APEX. Adv. Space Res. 13, 103-111. doi: 10.1016/0273-1177(93)9 0057-I

Podgorny, I. M. (1982). Active experiments in space, laboratory experiments and numerical simulation. J. Geomag. Geoelectr. 34, 53-92. doi: 10.5636/jgg.34.53

Prech, L. (1995). Active Experiments in the Earth Magnetosphere: Project APEX. Ph.D. thesis, Charles University, Prague. in Czech.

Prech, L. (2002). Active Experiments in the Earth Magnetosphere. Habilitation thesis, Charles University, Prague. in Czech.

Prech, L., Nemecek, Z., and Safrankova, J. (1998). Observations of the beamplasma interaction during the APEX artificial electron beam emission. Adv. Space Res. 21, 723-728.

Prech, L., Nemecek, Z., Safrankova, J., and Omar, A. (2002). Actively produced high-energy electron bursts within the magnetosphere: the APEX project. Ann. Geophys. 20, 1529-1538. doi: 10.5194/angeo-20-152 9-2002

Prech, L., Nemecek, Z., Safrankova, J., Simunek, J., and Smilauer, J. (1999). Artificial ion beam effects on spacecraft potential. Adv. Space Res. 24, 1027 1032. doi: 10.1016/S0273-1177(99)00557-8

Prech, L., Nemecek, Z., Safrankova, J., Simunek, J., Truhlik, V., and Shutte, N. (1995). Response of the electron-energy distibution to an artificially emitted electron beam - APEX experiment. Adv. Space Res. 15, 33-36. doi: 10.1016/0273-1177(95)00007-2

Pritchett, P. L. (1991). A three-dimensional simulation model for electron beam injection experiments in space. J. Geophys. Res. 96, 13781-13793. doi: 10.1029/91JA01266

Raitt, W. J. (1995). "Stimulating our piece of universe: Active experiments," in Rev. Geophys. Suppl., U.S. National Report to International Union of Geodesy and Geophysics 1991-1994, pages 559-564.

Rothkaehl, H., and Klos, Z. (1996). HF radio emissions as a tool of ionospheric plasma diagnostics. Ann. Geofis. 39, 833-838.

Rothkaehl, H., Klos, Z., Dokukin, V. S., and Triska, P. (1995). Remote spacecraft observations of waves excited by the pulse electron beam injected into ionosphere. Adv. Space Res. 15, 25-28. doi: 10.1016/0273-1177(95) 00005-Y

Ruzhin, Y. Y., and Vaskov, V. V. (1992). "Hybrid active experiments with artificial injections in space," in APEX Project. Scientific Purposes, Simulation, Technique and Equipment of Experiment, eds V. N. Oraevsky and Y. Y. Ruzhin (Moscow: Nauka), 54-67. in Russian.

Sagdeev, R. Z., Managadze, G. G., Mayorov, A. D., Lyakhov, S. B., Martinson, A. A., Romanovsky, Y. A., et al. (1981). Peculiarities of the environment disturbance during the electron beam injection from the rocket. Adv. Space Res. 1, 77-87.

Santolik, O., and Parrot, M. (2006). "Propagation analysis of electromagneticwaves: application to auroral kilometric radiation," in Geospace Electromagnetic Waves and Radiation, Vol. 687 of Lecture Notes in Physics, eds J. W. LaBelle and R. A. Treumann (Berlin: Springer), 297-312.

Santolik, O., Parrot, M., and Lefeuvre, F. (2003). Singular value decomposition methods for wave propagation analysis. Radio Sci. 38:1010. doi: 10.1029/2000RS002523

Santolik, O., Parrot, M., and Nemec, F. (2016). Propagation of equatorial noise to low altitudes: decoupling from the magnetosonic mode. Geophys. Res. Lett. 43, 6694-6704. doi: 10.1002/2016GL069582 
Taubenschuss, U., and Santolik, O. (2018). Wave polarization analyzed by singular value decomposition of the spectral matrix in the presence of noise. Surveys Geophys. 39, 1-31. doi: 10.1007/s10712-018-9496-9

Triska, P., Jiricek, F., Velicky, V., and Vojta, J. (1990). A subsatellite for motherdaughter active space experiments. Adv. Space Res. 10, 165-168.

Volokitin, A. S., Ruzhin, Y. Y., Korobeynikov, V. G., and Dokukin, V. S. (2000). Magnetic effects of plasma beam injection in ionosphere (experiment APEX). Geomagn. Aeron. 40, 133-137. in Russian.

Watermann, J., Wilhelm, K., Torkar, K. M., and Riedler, W. (1988). Spacelab-1 observations of suprathermal electrons induced by artificial electron beams. Adv. Space Res. 8, 111-114.

Winckler, J. R. (1980). The application of artificial electron beams to magnetospheric research. Rev. Geophys. Space Phys. 18, 659-682.

Zhang, X., Frolov, V., Zhou, C., Zhao, S., Ruzhin, Y., Shen, X., et al. (2016). Plasma perturbations HF-induced in the topside ionoshere. J. Geophys. Res. 121, 10052-10063. doi: 10.1002/2016JA022484
Zilavy, P., Prech, L., Nemecek, Z., and Safrankova, J. (2003). Spacecraft potential during an active experiment: a comparison of experimental results with a simple model. Ann. Geophys. 21, 915-922. doi: 10.5194/angeo-21915-2003

Conflict of Interest Statement: The authors declare that the research was conducted in the absence of any commercial or financial relationships that could be construed as a potential conflict of interest.

Copyright (c) 2018 Prech, Ruzhin, Dokukin, Nemecek and Safrankova. This is an open-access article distributed under the terms of the Creative Commons Attribution License (CC BY). The use, distribution or reproduction in other forums is permitted, provided the original author(s) and the copyright owner(s) are credited and that the original publication in this journal is cited, in accordance with accepted academic practice. No use, distribution or reproduction is permitted which does not comply with these terms. 